\title{
Coordinated Scheduling and Power Control in Cloud-Radio Access Networks
}

\author{
Ahmed Douik, Student Member, IEEE, Hayssam Dahrouj, Senior Member, IEEE, \\ Tareq Y. Al-Naffouri, Member, IEEE, and Mohamed-Slim Alouini, Fellow, IEEE
}

\begin{abstract}
This paper addresses the joint coordinated scheduling and power control problem in cloud-enabled networks. Consider the downlink of a cloud-radio access network (CRAN), where the cloud is only responsible for the scheduling policy, power control, and synchronization of the transmit frames across the single-antenna base-stations (BS). The transmit frame consists of several time/frequency blocks, called power-zones (PZ). The paper considers the problem of scheduling users to PZs and determining their power levels (PL), by maximizing the weighted sum-rate under the practical constraints that each user cannot be served by more than one base-station, but can be served by one or more power-zones within each base-station frame. The paper solves the problem using a graph theoretical approach by introducing the joint scheduling and power control graph formed by several clusters, where each is formed by a set of vertices, representing the possible association of users, BSs, and PLs for one specific PZ. The problem is, then, formulated as a maximumweight clique problem, in which the weight of each vertex is the sum of the benefits of the individual associations belonging to that vertex. Simulation results suggest that the proposed crosslayer scheme provides appreciable performance improvement as compared to schemes from recent literature.
\end{abstract}

Index Terms-Coordinated scheduling, power control, maximum-weight clique, joint scheduling and power control graph.

\section{OVERVIEW}

\section{A. Introduction}

The continuous increasing demand for high data rate services necessitates breakthroughs in network system architecture. With a progressive move towards full spectrum reuse and a positive trend in small-cell deployment, cloud-radio access networks (CRAN) become essential in large-scale interference management for next generation wireless systems (5G) [2]. Through its ability to allocate resources in a coordinated way across base-stations, cloud-enabled networks have the potential of mitigating inter-base-station interference through inter-basestation coordination.

Recent literature on CRAN assumes signal-level coordination and allows joint signal processing in the cloud [3]-[5].

A part of this paper [1] appears in proc. of the IEEE International Conference on Communication (ICC' 15), London, UK, June 2015.

Ahmed Douik is with the Department of Electrical Engineering, California Institute of Technology, Pasadena, CA 91125 USA (e-mail: ahmed.douik@caltech.edu).

Hayssam Dahrouj is with the Department of Electrical and Computer Engineering, Effat University, Jeddah 22332, Saudi Arabia (e-mail: hayssam.dahrouj@gmail.com).

T. Y. Al-Naffouri is with King Abdullah University of Science and Technology, Thuwal 23955-6900, Saudi Arabia, and also with King Fahd University of Petroleum and Minerals, Dhahran 31261, Saudi Arabia (e-mail: tareq.alnaffouri@kaust.edu.sa).

M.-S. Alouini is with the Division of Computer, Electrical and Mathematical Sciences and Engineering, King Abdullah University of Science and Technology, Thuwal 23955-6900, Saudi Arabia (e-mail: slim.alouini@kaust.edu.sa).
Such level of coordination allows joint beamforming, joint resource allocation, signal processing of data belonging to different users. However, signal-level coordination requires high-capacity links to share all data streams between all base-stations, and needs a substantial amount of backhaul communications. Furthermore, given the capacity limits of the backhaul links, substantial amount of backhaul/fronthaul communications may require sophisticated compression schemes. Designing the correlation matrices of the induced quantization noise becomes then a complicated process that is coupled with the determination of the beamforming vectors [3], [4]. This paper considers the CRAN problem from a different perspective, as it only considers scheduling and power control coordination at the cloud, which is more practical to implement, and at the same time allows base-stations to allocate the available radio resources efficiently. In the considered CRAN, basestations are connected to a central processor (cloud) which is responsible for scheduling users to base-stations resource blocks and determining the power levels of each resource blocks. Hence, only the scheduling policy and the power levels are communicated to the different base-stations which can be achieved through low-rate backhaul links resulting in a costefficient network design.

Different coordination strategies have been studied in the literature as a means to mitigate interference, namely the joint scheduling and power control [6]. Such joint resource allocation problem is mathematically challenging and so a decoupling approach has been proposed to solve each of the problems independently (e.g., iterating between determining the power levels for a fixed scheduling and finding the schedule for a fixed powers). The goal of this paper is to show that jointly considering the coordinated scheduling and power control problem, in a cloud-radio access network, provides appreciable performance improvement as compared to the conventional iterative approach.

Consider the downlink of a cloud-radio access network comprising several single-antenna base-stations (BSs) connected to one central processor (the cloud), which is only responsible for the scheduling policy, the synchronization, and the power level determination. The frame structure of every base-station consists of several time/frequency resource blocks called power-zones. Across the network, users are multiplexed across the power-zones under the constraint that each user cannot be connected to more than one base-station since, otherwise, signal-level coordination between base-stations is needed. Each user, however, can be connected to several power-zones belonging to the frame of one base-station. Further, each power-zone, which can be in practice seen as a generic term to denote time/frequency resource block of every BS, serves one and only one user. The coordinated 
scheduling problem becomes that of optimally scheduling users to base-stations and their power-zones and determining the optimal power levels used by each power-zone subject to the above practical constraints. A part of this paper considers the joint scheduling and power optimization problem with an objective of maximizing the weighted sum rate, where scheduling decisions are carried out by the cloud and relayed to the base-stations. Another part of the paper considers the coordinated scheduling problem alone (i.e., with fixed transmit power) with an objective to maximizing a generic utility function. Both parts of the paper solve the problems, i.e., the joint scheduling and power control, and the coordinated scheduling, using graph theory techniques.

\section{B. Related Work}

The problem of assigning users to base-stations is combinatorial in nature. Moreover, selecting the power levels for a fixed user schedule is a well-known non-convex problem. Therefore, the design of optimal solutions for the coordination problem is challenging, and a large amount of the literature considers user scheduling, and power allocation separately. The modular approach is more tractable and easier to implement.

In the past literature, scheduling is often performed on a per-base-station basis, given a pre-assigned association of users and base-stations, e.g., the classical proportionally fair scheduling [7], [8]. Unlike previous works where scheduling is performed with no inter-BS coordination, this paper considers the network-wide scheduling where coordination is carried by the cloud connecting the base-stations. The coordinated scheduling considered in this paper is particularly related to the concept developed in [9] in a soft-frequency reuse setup; however, the problem setup in [9] assumes an equal number of users and power-zones and boils down to a simple linear assignment problem, which can be solved using the classical auction methodology [10].

Inter-cell scheduling is specially investigated in [11]-[13]. While the authors in [11] consider maximizing the capacity of a multi-cell signal level coordinated network based on the complete co-channel gain information with a standard power control policy, the authors [12] consider a fully distributed scheme in which base-stations share the average channel state information only through low-rate backhaul communication. Through asymptotic analysis, the authors in [13] quantify the user diversity gain of their proposed inter-cell scheduling policy and derive bounds on the achievable ergodic capacity.

In the aforementioned works, the power levels used by each base-station is assumed to be fixed. To further account for the power optimization step, papers [14]-[16] consider updating the power in an outer step, i.e., for fixed scheduling. The authors in [17] present an alternative strategy which uses a dynamic interference avoidance scheme to mitigate excessive inter-cell interference. Reference [18] provides asymptotic rate-maximization properties of the power control and scheduling in multi-cell networks.

The joint scheduling and power control problem becomes an active research area of interest of late, e.g., [19]-[22]. However, such references only consider multi-cell OFDMA networks with negligible cell-to-cell interference. Other works on the joint optimization problem only guarantee convergence to a stationary point of the overall optimization problem, e.g., the work in [23] which proposes a branch-and-bound algorithm to maximize the number of served users under rate constraints.

\section{Contribution}

This paper considers the joint scheduling and power control problem by maximizing the weighted sum rate subject to network connectivity constraints, i.e., the problem of scheduling users to power-zones (PZ) and determining their power levels (PL) in a coordinated fashion across the network. Unlike previous works that consider iterating between solving the scheduling problem for fixed transmit power and optimizing the power for a given schedule, this paper proposes solving the joint optimization problem. Scheduling is performed under the practical constraint that each user cannot be served by more than one base-station but can be served by one or more power-zone within each base-station frame. The paper main contribution is that it optimally solves the problem using a graph theoretical approach by introducing the joint scheduling and power control graph formed by several clusters, where every cluster is formed by a set of vertices, each representing the possible association of users, BSs, and PLs for one specific PZ. The problem is, then, formulated as a maximum-weight clique, in which the weight of each vertex is the sum of the benefits of the individual associations belonging to that vertex.

Further, the paper considers the coordinated scheduling problem only (i.e., under fixed transmit power) by maximizing a generic network-wide utility subject to the same connectivity constraint. The paper solves the problem by introducing the corresponding scheduling graph in which each vertex represents a single association of one user, one BS, and one PZ.

The above-considered problems are shown to be equivalent to maximum-weight clique problems, which can be globally solved using efficient algorithms [24], [25]. Simulation results suggest that the proposed cross-layer scheme outperforms iterative schemes from the literature and provide a significant gain, especially at high interference regime.

The rest of this paper is organized as follows: In Section II, the system model and the problem formulation are presented. Section III shows the optimal solution to the joint scheduling and power control problem. In Section IV, the optimal solution to the scheduling problem under fixed transmit power is investigated. Simulation results are presented in Section V and the paper concluded in Section VI.

\section{Notations}

Let $|\mathcal{X}|$ denotes the cardinality of a set $\mathcal{X}$ and $\mathcal{P}(\mathcal{X})$ its power set. The set denoted by $\mathcal{A} \times \mathcal{B}$ represents the Cartesian product of the two sets $\mathcal{A}$ and $\mathcal{B}$. Finally, let $\delta($.$) be the discrete$ Dirac function, i.e. $\delta(x)$ is 1 if $x=0$, and $\delta(x)$ is 0 if $x \neq 0$. The notation $\{x\}$ denotes a set containing the single element $x$ and $(x, y)$ denotes the real interval between $x \leq y$. 


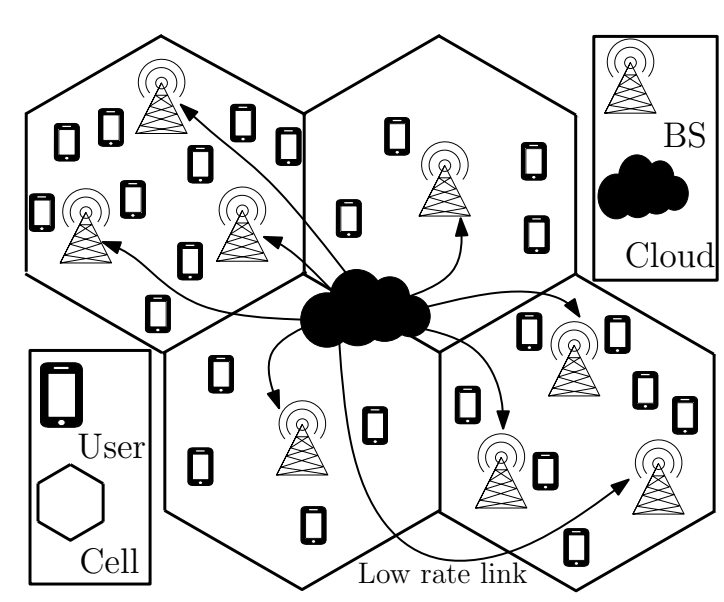

Fig. 1. Cloud enabled network composed of 8 base-stations and 24 users. Only low-rate backhaul links are required to connect the cloud to the multiple base-stations.

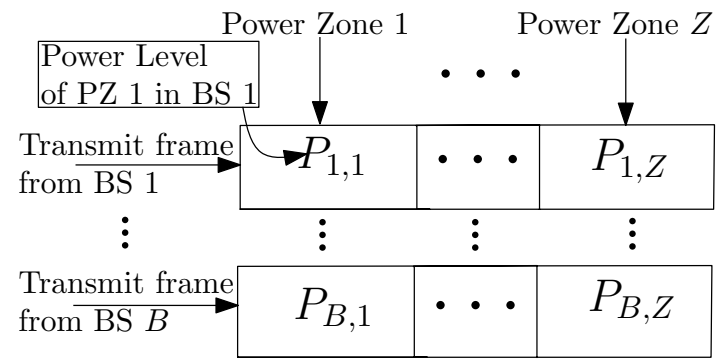

Fig. 2. Frame structure of $B$ base-stations each containing $Z$ power-zones.

\section{System Model and Problem Formulation}

\section{A. System Model}

Consider the downlink of a cloud radio wireless network of $B$ single-antenna BSs connected to a central cloud through low-rate backhaul links and serving $U$ users in total, as shown in Figure 1, which shows a CRAN formed by $B=8$ BSs and $U=25$ users. Let $\mathcal{B}$ be the set of all BSs in the system and $\mathcal{U}$ be the set of all users. The frame of each base-station consists of $Z$ time/frequency resource blocks (called herein power-zones). Let $\mathcal{Z}$ be the set of PZs of the frame of a BS. As shown in Figure 2, the paper assumes orthogonal PZs, i.e., interference is only seen from power-zones with the same index $z$ across the different base-stations. The total number of available PZs is $Z_{\text {tot }}=B \times Z$. The power level of each PZ $z$ in BS $b$ 's frame is denoted by $P_{b z}$, as shown in Figure 2. The value of $P_{b z}$ is called the power levels (PLs) of BS $b$ and PZ $z$. This paper assumes individual $\mathrm{PZ}$ constraint $P_{b z} \leq P_{b z}^{\max }$. Let $\mathbf{P}$ be a $B \times Z$ matrix containing the PLs of all PZs in the BSs. In other words, $\mathbf{P}=\left[P_{b z}\right]$.

The cloud, connecting the different BSs, guarantees that the transmission of the different frames is synchronized across all BSs. The cloud is further responsible for determining the scheduling and power control policies of the network. Let $h_{b z}^{u} \in \mathbb{C}, \forall(u, b, z) \in \mathcal{U} \times \mathcal{B} \times \mathcal{Z}$ be the channel from the $b$ th BS to user $u$ when user $u$ is assigned to $\mathrm{PZ} z$. The paper considers general channels without restrictions on their distributions. However, assumes that the values of the channel gains are perfectly known as the cloud. The corresponding signal-to-interference plus noise-ratio (SINR) is:

$$
\operatorname{SINR}_{b z}^{u}(\mathbf{P})=\frac{P_{b z}\left|h_{b z}^{u}\right|^{2}}{\Gamma\left(\sigma^{2}+\sum_{b^{\prime} \neq b} P_{b^{\prime} z}\left|h_{b^{\prime} z}^{u}\right|^{2}\right)},
$$

where $\sigma^{2}$ is the Gaussian noise variance and $\Gamma$ denotes the SINR gap.

Remark 1. Note that the $\operatorname{SINR}_{b z}^{u}(\mathbf{P})$ depends only on the scheduled users in $P Z z$ and the corresponding PLs $P_{b^{\prime} z}$ but does not depend on users scheduled in other $P Z z^{\prime} \neq z$ or their corresponding PLs $P_{b^{\prime}} z^{\prime}$.

\section{B. Problem Formulation}

This paper considers the problem of assigning users to PZs in BSs under the following constraints:

- C1: Each user can connect at most to one BS but possibly to several PZs in that BS.

- C2: Each PZ is allocated to exactly one user.

- C3: Each PL is bounded by a nominal maximal value.

A part of this paper considers the weighted sum-rate maximization problem where the rate of user $u$ when assigned to the $z$ th PZ of the $b$ th BS is weighted by a factor $a_{u b z}$, which is fixed throughout this paper. Such factor can typically be adjusted in an outer loop for load balancing and fairness purposes. However, this falls outside the scope of the current paper. Let $X_{u b z}$ be a binary variable that is set to 1 if user $u$ is mapped to the $z$ th $\mathrm{PZ}$ of the $b$ th $\mathrm{BS}$, and zero otherwise. Further, let $Y_{u b}$ be a binary variable that is set to 1 if user $u$ is mapped to the $b$ th BS, and zero otherwise. Next section considers the following network-wide optimization problem:

$$
\begin{aligned}
\max & \sum_{u, b, z} a_{u b z} X_{u b z} \log _{2}\left(1+\operatorname{SINR}_{b z}^{u}(\mathbf{P})\right) \\
\text { s.t. } & Y_{u b}=\min \left(\sum_{z} X_{u b z}, 1\right), \forall(u, b) \in \mathcal{U} \times \mathcal{B}, \\
& \sum_{b} Y_{u b} \leq 1, \quad \forall u \in \mathcal{U}, \\
& \sum_{u} X_{u b z}=1, \quad \forall(b, z) \in \mathcal{B} \times \mathcal{Z}, \\
& 0 \leq P_{b z} \leq P_{b z}^{\max }, \forall(b, z) \in \mathcal{B} \times \mathcal{Z}, \\
& X_{u b z}, Y_{u b} \in\{0,1\}, \forall(u, b, z) \in \mathcal{U} \times \mathcal{B} \times \mathcal{Z},
\end{aligned}
$$

where the optimization is over the binary variables $X_{u b z}, Y_{u b}$, and the continuous variables $P_{b z}, \forall(u, b, z) \in \mathcal{U} \times \mathcal{B} \times \mathcal{Z}$. Here the constraints in (2b) and (2c) correspond to constraint $\mathrm{C} 1$, the equality constraint in (2d) corresponds to constraint $\mathrm{C} 2$, and (2e) corresponds to constraint C3. Finding the global optimum to the mixed discrete and continuous optimization problem (2) may involve searching over all possible user-to-powerzone assignments, and determining the PLs for every possible assignment. This is clearly infeasible for any reasonably sized network.

The next section solves this joint coordinated scheduling and power control optimization problem by introducing the joint scheduling and power control graph formed by several clusters (called herein local power control graphs), where every cluster is formed by a set of vertices, each representing the possible association of users, BSs, and PLs for one specific $\mathrm{PZ}$. The problem is, then, formulated as a maximum-weight 
clique problem, in which the weight of each vertex is the sum of the benefits of the individual associations belonging to that vertex. Further, the paper proposes a solution to the particular problem in which PZs are maintained at fixed PLs. The resulting coordinated scheduling solution is then obtained by introducing the scheduling graph in which each vertex represents an individual association of one user, one PZ, and one BS.

\section{JoInt POWER CONTROL AND COORDINATED SCHEDULING}

In this section, the joint coordinated scheduling and power control optimization problem is solved by showing that it is equivalent to a maximum-weight clique problem, which can be globally solved using existing efficient solvers, e.g., [24], [25]. Note that the proposed joint scheduling and power control method presented in this section is centralized. All computation are carried out at the cloud and relayed to the BSs.

The section relies on the fact that the optimal solution of (2) can be reached by solving the problem for an intelligently constructed discrete set of PLs, i.e., replacing constraint (2e) by the constraint $P_{b z} \in \mathcal{P}$, where $\mathcal{P}$ is a set simultaneously constructed with the joint scheduling and power control graph. Hence, the first part of the section illustrates the optimal solution to the following optimization problem:

$$
\begin{aligned}
\max & \sum_{u, b, z} a_{u b z} X_{u b z} \log _{2}\left(1+\operatorname{SINR}_{b z}^{u}(\mathbf{P})\right) \\
\text { s.t. } & Y_{u b}=\min \left(\sum_{z} X_{u b z}, 1\right), \forall(u, b) \in \mathcal{U} \times \mathcal{B}, \\
& \sum_{b} Y_{u b} \leq 1, \quad \forall u \in \mathcal{U}, \\
& \sum_{u} X_{u b z}=1, \quad \forall(b, z) \in \mathcal{B} \times \mathcal{Z}, \\
& P_{b z} \in \mathcal{P}, \forall(b, z) \in \mathcal{B} \times \mathcal{Z}, \\
& X_{u b z}, Y_{u b} \in\{0,1\}, \forall(u, b, z) \in \mathcal{U} \times \mathcal{B} \times \mathcal{Z},
\end{aligned}
$$

where the optimization is carried over the binary variables $X_{u b z}$ and $Y_{u b}$ and the discrete variable $P_{b z} \in \mathcal{P}$.

In the first subsection, the optimization problem (3) is rewritten in a more insightful form which allows it to be optimally solved, as shown in the second and third subsections. The philosophy of the proposed solution is to use clustering of vertices in a well-defined graph called local power control graph or cluster. Each cluster represents an index of a PZ in the network (across the multiple BSs). Inside each cluster, vertices are not connected. However, connections may exist between vertices belonging to different clusters, depending on the schedule feasibility. The graph containing the $Z$ clusters is called the joint scheduling and power control graph. The problem is, then, shown to be a maximum-weight clique problem among the cliques of size $Z$.

In the fourth subsection, the solution of the original optimization problem (2) is shown to be achieved using the method developed for (3) for specific and well constructed set $\mathcal{P}$ of PLs. The last subsection characterizes the complexity of the optimal solution of (2).

\section{A. Problem Reformulation}

Let $\mathcal{A}$ be the set of all possible associations between users, base-stations, power-zones, and power levels i.e. $\mathcal{A}=\mathcal{U} \times \mathcal{B} \times$ $\mathcal{Z} \times \mathcal{P}$. Define $\varphi_{u}$ as the mapping function from the set $\mathcal{A}$ to the set of users $\mathcal{U}$, i.e., $\varphi_{u}(y)=u, \quad \forall y=(u, b, z, p) \in \mathcal{A}$. In other words, for each association $y \in \mathcal{A}$, the function $\varphi_{u}$ returns the index of the user considered in the association. In a similar way, we define $\varphi_{b}(y)=b, \varphi_{z}(y)=z$ and $\varphi_{p}(y)=$ $p, \forall y=(u, b, z, p) \in \mathcal{A}$. In other words, for each association, the functions $\varphi_{b}, \varphi_{z}$ and $\varphi_{p}$ return the index of the BS, PZ, and PL, respectively, considered in the association.

The power-set $\mathcal{P}(\mathcal{A})$, representing all possible associations between users, base-stations, power-zones, and power levels is also the set of all schedules, regardless if the schedules satisfy the constraints $\mathrm{C} 1, \mathrm{C} 2$, and $\mathrm{C} 3$ or not. The set of all feasible schedules can then be characterized as a function of the individual schedules as outlined in the following lemma.

Lemma 1. $\mathcal{F}$, the set of schedules that satisfy constraints $C 1$, $C 2$, and $C 3$, can mathematically be defined as follows:

$$
\begin{aligned}
& \mathcal{F}=\left\{\mathbf{S} \in \mathcal{P}(\mathcal{A}) \text { such that } \forall s \neq s^{\prime} \in \mathbf{S}\right. \\
& \delta\left(\varphi_{u}(s)-\varphi_{u}\left(s^{\prime}\right)\right) \varphi_{b}(s)=\varphi_{b}\left(s^{\prime}\right) \delta\left(\varphi_{u}(s)-\varphi_{u}\left(s^{\prime}\right)\right), \\
& \left(\varphi_{b}(s), \varphi_{z}(s)\right) \neq\left(\varphi_{b}\left(s^{\prime}\right), \varphi_{z}\left(s^{\prime}\right)\right), \\
& \left.|\mathbf{S}|=Z_{\text {tot }}\right\} .
\end{aligned}
$$

Proof: The proof can be found in Appendix A.

Define the function $\mathfrak{g}$ from $\mathcal{A} \times \mathcal{F}$ to $\mathbb{R}$ as the benefit of each individual association $s$ and the schedule $\mathbf{S}$, i.e.: $g(s, \mathbf{S})=$ $\log _{2}\left(1+\operatorname{SINR}_{b z}^{u}(\mathbf{P})\right)$, where $(u, b, z)$ is the tuple corresponding to the association $s$, i.e. $(u, b, z)=\left(\varphi_{u}(s), \varphi_{b}(s), \varphi_{z}(s)\right)$ and $\mathbf{P}$ is the PLs matrix corresponding to the schedule $\mathbf{S}$, i.e., $\mathbf{P}=\left[P_{b^{\prime} z^{\prime}}\right]$ such that $\left(b^{\prime} z^{\prime}\right)=\left(\varphi_{b}\left(s^{\prime}\right), \varphi_{z}\left(s^{\prime}\right)\right)$ and $P_{b^{\prime} z^{\prime}}=\varphi_{p}\left(s^{\prime}\right), \forall s^{\prime} \in \mathbf{S}$. Let $a(s)$ be the weight of the association $s$, i.e., $a(s)=a_{u b z}$ where $s=(u, b, z, p)$.Therefore, the optimization problem (3) can be written as:

$$
\begin{array}{ll}
\max & \sum_{s \in \mathbf{S}} a(s) \mathfrak{g}(s, \mathbf{S}) \\
\text { s.t. } & \mathbf{S} \in \mathcal{F},
\end{array}
$$

\section{B. Local Power Control Graph}

In this section, we introduce the local graph called local power control graph. Note that this entire graph is seen as a single cluster in the joint scheduling and power control graph that is introduced in the next subsection.

For each index $z$ of a $\mathrm{PZ}$ in the network, such graph is created. Note that throughout the paper, the words local power control graph and cluster are used interchangeably. We consider in this subsection, the generation of such graph for PZs indexed by $z$. Hence the associations that are considered in the generation of the graph are $s \in \mathcal{A}$ such that $\varphi_{z}(s)=z$.

Let $\mathcal{S}^{z}$ be the set of all schedules associated with $\mathrm{PZ} z$ (feasible or not) defined as follows:

$$
\mathcal{S}^{z}=\{\mathcal{U}, \mathcal{B}, z, \mathcal{P}\}^{B}
$$

From the feasibility condition $\mathrm{C} 2$ of a schedule, each PZ should be connected to exactly one user. Since there are $B$ BSs in the network, then there are $B$ PZs indexed by $z$. Hence, for 
each index $z \in \mathcal{Z}$, there exist precisely $B$ associations, which explains the existence of $B$ associations in the definition of $\mathcal{S}^{z}$ (6). For simplicity of notations, we assume that the $i$ th association in any element of $\mathcal{S}^{z}$, call it $\mathbf{S}$, is associated with the $i$ th $\mathrm{BS}$ in the network. For example, the first association of the schedule $\mathbf{S} \in \mathcal{S}^{z}$ is $(u, 1, z, p)$.

Given that each user cannot connect to multiple BSs, the same user cannot appear more than once in a feasible schedule. Similarly, the same BS cannot appear more than one for the same reason. Let $\mathcal{F}^{z}$ be the set of the feasible associations for PZs indexed by $z$ defined by:

$$
\begin{array}{r}
\mathcal{F}^{z}=\left\{\mathbf{S}^{z} \in \mathcal{S}^{z}\right. \text { such that } \\
\varphi_{u}(s) \neq \varphi_{u}\left(s^{\prime}\right), \forall s \neq s^{\prime} \in \mathbf{S}^{z} \\
\left.\varphi_{b}(s) \neq \varphi_{b}\left(s^{\prime}\right), \forall s \neq s^{\prime} \in \mathbf{S}^{z}\right\}
\end{array}
$$

Let $\mathcal{G}^{z}\left(\mathcal{V}^{z}, \mathcal{E}^{z}\right)$ be the local power control graph. This graph is generated by creating a vertex $v^{z} \in \mathcal{V}^{z}$ for each possible association $\mathbf{S}^{z} \in \mathcal{F}^{z}$. This cluster does not contain any connection, i.e., $\mathcal{E}^{z}=\varnothing$. The weight of each vertex is the weighted sum rate of the represented association. The weight of vertex $v^{z}$ associated with the schedule $\mathbf{S}^{z}$ is:

$$
w\left(v^{z}\right)=\sum_{s \in \mathbf{S}^{z}} a(s) \mathfrak{g}\left(s, \mathbf{S}^{z}\right) .
$$

\section{Construction of Joint Scheduling and Power Control Graph}

To construct the joint scheduling and power control graph $\mathcal{G}(\mathcal{V}, \mathcal{E})$, we first generate all the clusters for PZs $z, z \in \mathcal{Z}$. The set of vertices of the joint scheduling and power control graph is the union of all the vertices in all local power control graphs generated in the previous subsection. In other words, we have $\mathcal{V}=\bigcup_{z \in \mathcal{Z}} \mathcal{V}^{z}$. Two vertices $v^{z_{1}} \in \mathcal{G}^{z_{1}}$ and $v^{z_{2}} \in \mathcal{G}^{z_{2}}$ belonging to distinct clusters $z_{1} \neq z_{2}$ are connected if their combination results in a feasible schedule. In other words, they are connected if the users they represents are not connected to multiple BSs. In mathematical terms, let $v^{z_{1}} \in \mathcal{G}^{z_{1}}$ associated with the schedule $\mathbf{S}^{z_{1}}$ and $v^{z_{2}} \in \mathcal{G}^{z_{2}}$ associated with the schedule $\mathbf{S}^{z_{2}}$ with $z_{1} \neq z_{2}$. The vertices $v^{z_{1}}$ and $v^{z_{2}}$ are connected if:

$$
\begin{aligned}
& \delta\left(\varphi_{u}\left(s^{z_{1}}\right)-\varphi_{u}\left(s^{z_{2}}\right)\right) \delta\left(\varphi_{b}\left(s^{z_{1}}\right)-\varphi_{b}\left(s^{z_{2}}\right)\right) \\
& =\delta\left(\varphi_{u}\left(s^{z_{1}}\right)-\varphi_{u}\left(s^{z_{2}}\right)\right), \forall\left(s^{z_{1}}, s^{z_{2}}\right) \in \mathbf{S}^{z_{1}} \times \mathbf{S}^{z_{2}}
\end{aligned}
$$

Consider the graph $\mathcal{G}(\mathcal{V}, \mathcal{E})$ as constructed above. Then, define $\mathcal{C}$ as the set of all possible cliques with degree $Z$. The problem (3) can then be written as a maximum-weight clique problem, as highlighted in the following theorem.

Theorem 1. The problem of associating users to power-zones and selecting the PL for each PZ (3) can be written as:

$$
\begin{aligned}
\mathbf{S}^{*} & =\arg \max _{\mathbf{S} \in \mathcal{F}} \sum_{s \in \mathbf{S}} a(s) \mathfrak{g}(s, \mathbf{S}) \\
& =\arg \max _{\mathbf{C} \in \mathcal{C}} \sum_{v \in \mathbf{C}} w(v),
\end{aligned}
$$

where $\mathbf{C} \in \mathcal{C}$ is a clique in the joint scheduling and power control graph, $\mathcal{C}$ is the set of all possible cliques of degree $Z$, and $w(v)$ is the weight of each corresponding vertex defined in (7). In other words, the optimal solution of the coordinated scheduling problem (3) is the maximum-weight clique of degree $Z$.
Proof: The proof can be found in Appendix B maximum-weight clique problems are NP-hard problems, in general. There exist, however, efficient algorithms to solve the problem; see [24], [25] and references therein.

\section{Optimal Power Control and Assignment Solution}

In this subsection, we provide an efficient method to reach the optimal solution of the original optimization problem (2), using the results developed for the optimization problem (3). We show that for a well constructed discrete set of PLs, we can achieve the optimal solution of the joint scheduling and power optimization problem. Define, for $\mathrm{PZ} z$, the set of all $(u, b, z)$ associations $\tilde{\mathcal{S}}^{z}$ and feasible schedules $\tilde{\mathcal{F}}^{z}$ for a cluster $\mathcal{G}^{z}$ by omitting the PLs as follows:

$$
\begin{aligned}
& \tilde{\mathcal{S}}^{z}=\{\mathcal{U}, \mathcal{B}, z\}^{B} \\
& \tilde{\mathcal{F}}^{z}=\left\{\tilde{\mathbf{S}} \in \tilde{\mathcal{S}}^{z}\right. \text { such that } \\
& \quad \varphi_{u}(\tilde{s}) \neq \varphi_{u}\left(\tilde{s}^{\prime}\right), \forall \tilde{s} \neq \tilde{s}^{\prime} \in \tilde{\mathbf{S}} \\
& \left.\quad \varphi_{b}(\tilde{s}) \neq \varphi_{b}\left(\tilde{s}^{\prime}\right), \forall \tilde{s} \neq \tilde{s}^{\prime} \in \tilde{\mathbf{S}}\right\} .
\end{aligned}
$$

For a fixed schedule $\tilde{\mathbf{S}}=\left\{\tilde{s}_{1}, \cdots, \tilde{s}_{B}\right\} \in \tilde{\mathcal{F}}^{z}$, the optimal PLs $\left(p_{1 z}^{*}, \cdots, p_{B z}^{*}\right)$ that maximize the weighted sum rate are the solution to the following optimization problem:

$$
\begin{aligned}
& \max _{p_{b z}} \sum_{b \in \mathcal{B}} a\left(\tilde{s}_{b}\right) \mathfrak{g}\left(\tilde{s}_{b}, \tilde{\mathbf{S}}\right) \\
& \text { s.t. } 0 \leq p_{b z} \leq P_{b z}^{\max }, \forall b \in \mathcal{B},
\end{aligned}
$$

where the optimization is over the power levels $p_{b z}, \forall b \in \mathcal{B}$. The optimization problem (11) is a well known non-convex problem [26], [27]. Despite the non-convexity of the problem, it can be solved efficiently. In [26], the authors propose an algorithm to achieve the optimal solution of (11). Other efficient algorithms (e.g., [27]-[30]) can be used but may only lead to a local optimal solution to the problem. Our scheme can make use of any of such power optimization algorithms to solve problem (11).

In order to solve the joint scheduling and power optimization problem, we first generate the joint scheduling and power control graph as follows: for each $\mathrm{PZ} z \in \mathcal{Z}$, a schedule $\tilde{\mathbf{S}} \in \tilde{\mathcal{F}}^{z}$ is created for all possible associations. The optimal PLs of each association are, then, computed by solving the optimization problem (11). The vertex in the local power control graph $\mathcal{G}^{z}$ is generated by appending the calculated PLs to the schedule $\widetilde{\mathbf{S}}$ whose PLs are omitted as shown in (10). The process is repeated for all PZs $z \in \mathcal{Z}$. The joint scheduling and power control graph $\mathcal{G}$ is generated afterwards by merging all the clusters and adding connections according to condition (8).

The optimal solution to the joint scheduling and power optimization problem is, therefore, obtained by solving the maximum-weight clique problem in $\mathcal{G}$ in which the weight of each vertex is computed using (7). The entire algorithm structure is provided in Algorithm 1. For illustration, the graph $\mathcal{G}$ resulting from Algorithm 1 for a network composed of 2 base-stations, 2 power-zones per base-station and 3 users is shown in Figure 3. The following theorem characterizes the solution reached by Algorithm 1:

Theorem 2. The optimal solution to the joint scheduling and power optimization problem (2) is achieved using Algorithm 1. 


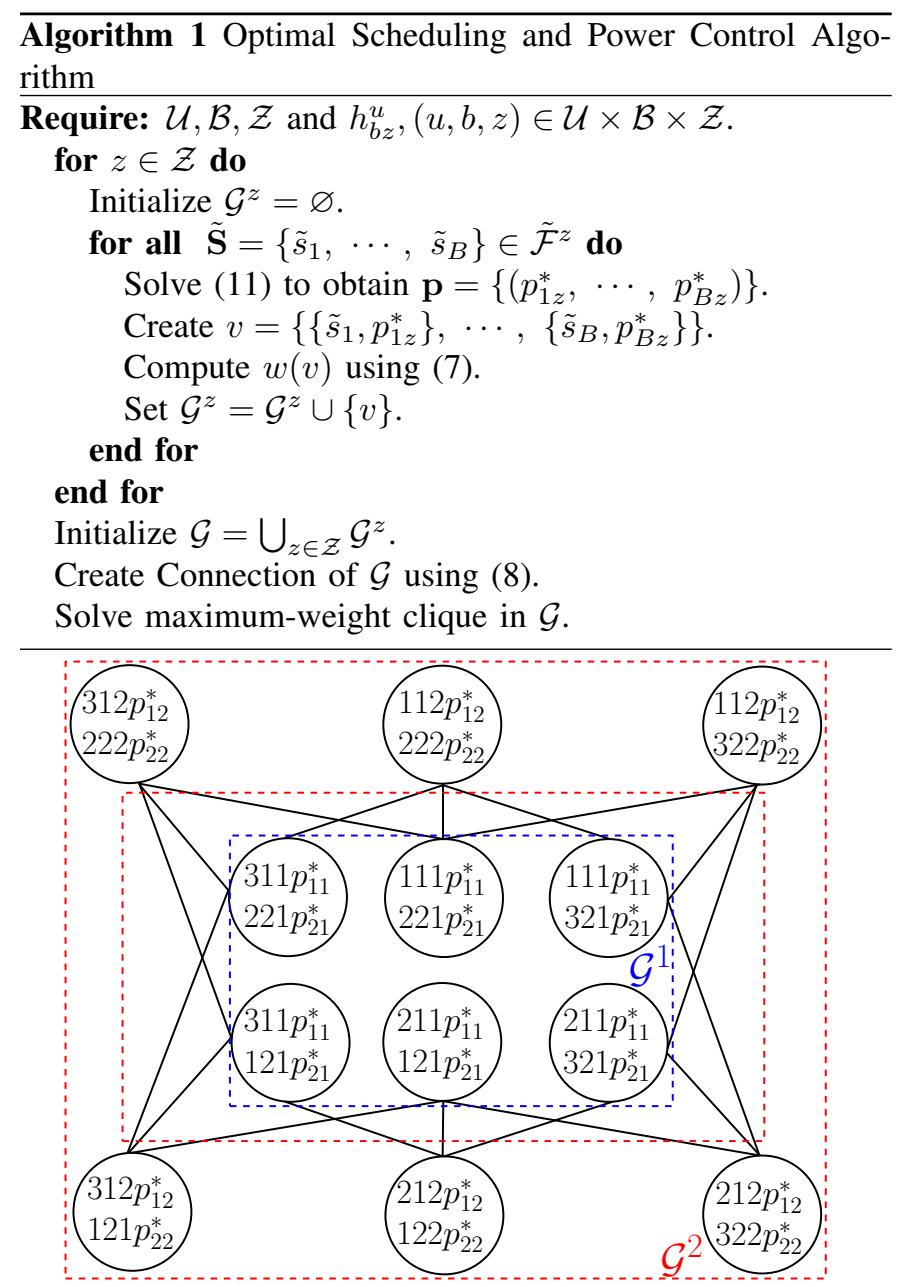

Fig. 3. Joint scheduling and power control graph for a network composed of of 2 base-stations, 2 power-zones per base-station and 3 users.

Proof: The proof of can be found in Appendix C

\section{E. Complexity Analysis}

From Theorem 2, we clearly see that the joint scheduling and power optimization problem is equivalent to a maximumweight clique, which is an NP-hard problem, and so is the problem (2). The following lemma characterizes the complexity of reaching the optimal solution of (2) using Algorithm 1.

Lemma 2. The complexity of reaching the optimal solution of (2) using Algorithm 1 is $\left({ }^{U} P_{B}\right) \cdot Z \cdot c(B)+f\left(\left({ }^{U} P_{B}\right) \cdot Z\right)$, where ${ }^{n} P_{r}=\frac{n !}{(n-r) !}$ is the number of permutations, $c(x)$ is the complexity of solving (11) with $x$ variables and $f(x)$ is the complexity of solving the maximum-weight clique in a graph containing $x$ vertices.

Proof: The proof of can be found in Appendix D

\section{Coordinated Scheduling}

Most of the used algorithms in the literature to solve the scheduling and power control problem rely on the decoupling of the variables and iterating between the scheduling and the power control problems. In other words, for a fixed scheduling, the PLs are determined then, for fixed PLs, the schedule is determined. Iteration between these two problems is carried out until convergence. For a specified user scheduling, the power control problem can be optimally solved as shown in (11). The optimal solution for the coordinated scheduling problem, i.e., for fixed PLs, is, however, not considered in the past literature. Towards that goal, this sections addresses the coordinated scheduling problem by maximizing a generic network utility and shows that the problem can be formulated as a maximum-weight clique problem which can be solved efficiently.

Let $P_{b z}, \forall(b, z) \in \mathcal{B} \times \mathcal{Z}$ be the PL of the $b$ th BS and the $z$ th PZ maintained at a fixed level. Further, let $r_{u b z}$ be a generic network-wide utility of assigning user $u$ to $\mathrm{BS} b$ and $\mathrm{PZ} z$. The problem can be written as:

$$
\begin{aligned}
\max & \sum_{u, b, z} r_{u b z} X_{u b z} \\
\text { s.t. } & Y_{u b}=\min \left(\sum_{z} X_{u b z}, 1\right), \forall(u, b) \in \mathcal{U} \times \mathcal{B}, \\
& \sum_{b} Y_{u b} \leq 1, \quad \forall u \in \mathcal{U}, \\
& \sum_{u} X_{u b z}=1, \quad \forall(b, z) \in \mathcal{B} \times \mathcal{Z}, \\
& X_{u b z}, Y_{u b} \in\{0,1\}, \forall(u, b, z) \in \mathcal{U} \times \mathcal{B} \times \mathcal{Z},
\end{aligned}
$$

where the optimization is over the binary variables $X_{u b z}$, $Y_{u b}, \forall(u, b, z) \in \mathcal{U} \times \mathcal{B} \times \mathcal{Z}$, where the constraints in (12b) and $(12 \mathrm{c})$ correspond to constraint $\mathrm{C} 1$, where the equality constraint in (12d) corresponds to constraint $\mathrm{C} 2$.

The solution hinges on the fact that the problem (12) can be reformulated as a maximum-weight clique problem. The section first shows how to build the corresponding scheduling graph, and then reformulates the problem. It also presents efficient heuristic to solve the scheduling problem. Note that, similar to the previous section, the proposed scheduling method presented in this section is centralized in nature. The scheduling solutions are carried out by the centralized processor at the cloud and coordinated to the base-stations.

\section{A. Construction of Scheduling Graph}

Let $\mathcal{A}$ be the set of all possible associations between users, base-stations, and power-zones, i.e. $\mathcal{A}=\mathcal{U} \times \mathcal{B} \times \mathcal{Z}$. The scheduling graph $\mathcal{G}(\mathcal{V}, \mathcal{E})$ can then be constructed as follows: Generate a vertex $v$ for all possible associations $s \in \mathcal{A}$. Two distinct vertices $v_{1}$ and $v_{2}$ in $\mathcal{V}$ are connected by an edge in $\mathcal{E}$ if the following conditions hold:

- C1: if $\varphi_{u}\left(v_{1}\right)=\varphi_{u}\left(v_{2}\right)$ then $\varphi_{b}\left(v_{1}\right)=\varphi_{b}\left(v_{2}\right)$ : this condition states that the same user cannot connect to multiple BSs.

- $\mathrm{C} 2:\left(\varphi_{b}\left(v_{1}\right), \varphi_{z}\left(v_{1}\right)\right) \neq\left(\varphi_{b}\left(v_{2}\right), \varphi_{z}\left(v_{2}\right)\right)$ : this constraint states that two different users cannot be connected to the same PZ.

Figure 4 shows an example of the scheduling graph in a system with $U=2$ users, $B=2 \mathrm{BSs}$ and $Z=2 \mathrm{PZs}$. In this example, each vertex is labeled $u b z$, where $u, b$ and $z$ represent the indices of users, BSs and PZs respectively. We clearly see 


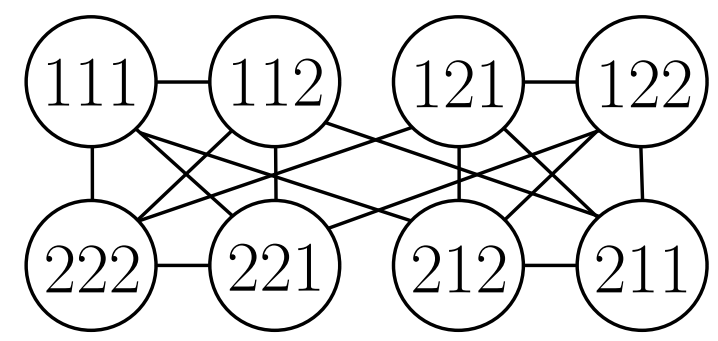

Fig. 4. Example of scheduling graph for 2 users, 2 BSs and 2 PZs.

that the only possible cliques of size $Z_{\text {tot }}=B Z=4$ are $\{\{111,112,221,222\},\{121,122,211,212\}\}$.

\section{B. Optimal Assignment Solution}

Consider the scheduling graph $\mathcal{G}(\mathcal{V}, \mathcal{E})$ associated with the constraints $\mathrm{C} 1$ and $\mathrm{C} 2$, as constructed in subsection IV-A. Then, define $\mathcal{C}$ as the set of all possible cliques with degree $Z_{\text {tot. }}$. The problem (12) can then be written as a maximumweight clique problem, as highlighted in the following theorem.

Theorem 3. The scheduling problem of associating users to power-zones (12) can be written as:

$$
\begin{aligned}
\mathbf{S}^{*} & =\underset{\mathbf{S} \in \mathcal{F}}{\arg \max } \sum_{s \in \mathbf{S}} r(s) \\
& =\underset{\mathbf{C} \in \mathcal{C}}{\arg \max } \sum_{v \in \mathbf{C}} w(v),
\end{aligned}
$$

where $\mathbf{C} \in \mathcal{C}$ is a clique in the scheduling graph, and $w(v)$ is the weight of each vertex $v$. In other words, the optimal solution of the scheduling problem (12) is the maximum-weight clique of degree $Z_{\text {tot }}$ in the scheduling graph where the weight of each vertex $v \in \mathcal{V}$ associated with $s \in \mathcal{A}$ is defined as:

$$
w(v)=r(s) .
$$

Proof: The proof can be found in Appendix E

The complexity of reaching the optimal solution of (12) boils down to the complexity of solving the maximum-weight clique problem. In other words, the complexity of optimally solving the scheduling only problem is $f(U \cdot B \cdot Z)$, where $f(x)$ is the complexity of solving the maximum-weight clique in a graph containing $x$ vertices.

\section{Proposed Heuristic Algorithm}

Maximum weight cliques problems are NP-hard problems. For a graph $\mathcal{G}(\mathcal{V}, \mathcal{E})$, the complexity of solving the maximum weight clique problem is $\alpha^{|\mathcal{V}|}$ wherein $\mathcal{V}$ is the set of vertices and $1<\alpha<2$ is the complexity constant [24], [25], [31]. Even tough such complexity improves tremendously over the $|\mathcal{V}|^{2} 2^{|\mathcal{V}|}$ cost of generic binary program solvers, it is still prohibitive for moderate size networks. For example, using the globally convergent algorithm provided in [31], the complexity of the proposed solution is $1.21^{U B Z}$.

To solve the maximum-weight clique problem in linear time with the problem size, a simple heuristic is proposed in this subsection. First, construct the graph $\mathcal{G}$. The idea here is to update the schedule $\mathbf{S}$ sequentially by adding the vertex with

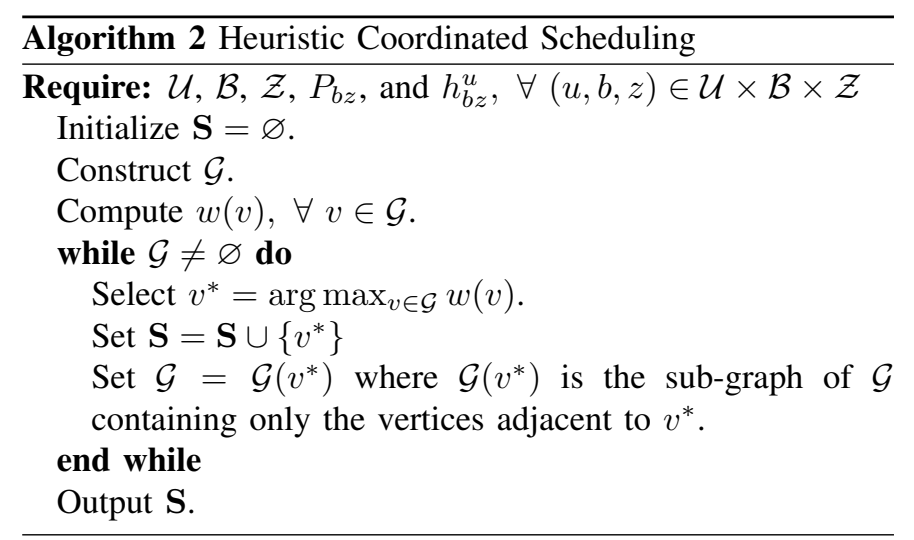

SYSTEM MODEL PARAMETERS

\begin{tabular}{|c|c|}
\hline Channel Model & SUI-3 Terrain type B \\
\hline Channel Estimation & Perfect \\
\hline Background Noise Power & $-168.60 \mathrm{dBm} / \mathrm{Hz}$ \\
\hline SINR Gap $\Gamma$ & $0 \mathrm{~dB}$ \\
\hline Bandwidth & $10 \mathrm{MHz}$ \\
\hline
\end{tabular}

the highest weight at each step. Then, the graph is updated by removing all vertices not connected to the selected vertex, so as to guarantee that the constraints $\mathrm{C} 1$ and $\mathrm{C} 2$ are satisfied. The process is repeated until the graph becomes empty. The steps of the heuristic are summarized in Algorithm 2.

\section{Simulation Results}

This section shows the performance of the proposed algorithms in the downlink of a cloud-radio access network, similar to Figure 1. Users are uniformly placed in the network. The number of users, numbers of base-stations, number of powerzones per BS frame, and the maximum power $P^{\max }$ vary in the simulations so as to study the methods performance for various scenarios. Additional simulations parameters are summarized in Table I. The scheduling and power allocation schemes considered in this part are:

- Maximum power transmission in which the PLs are set to their maximum value $P^{\max }$.

- Iterative scheduling and power control in which the scheduling problem is solved for a fixed PLs. The PLs are then updated according to the new scheduling. Iteration between the two problems is carried until convergence.

- Optimal joint scheduling and power control using our proposed algorithm.

First, Figure 5 plots the sum-rate in $\mathrm{bps} / \mathrm{Hz}$ versus the number of users for a CRAN composed of 3 base-stations and 4 power-zones per frame. The figure shows that our proposed solution outperform the iterative approach. It further shows that the optimal scheduling outperforms the heuristic solution for all policies, particularly for large number of users. The gain is due to the fact that as the number of users increases, interference becomes larger, and so the role of coordinated scheduling and power control as an interference mitigation technique becomes more pronounced.

Figure 6 plots the sum-rate in $\mathrm{bps} / \mathrm{Hz}$ versus the number of power-zones per BS frame, for a CRAN composed of 3 basestations and 5 users. The optimal joint scheduling and power 


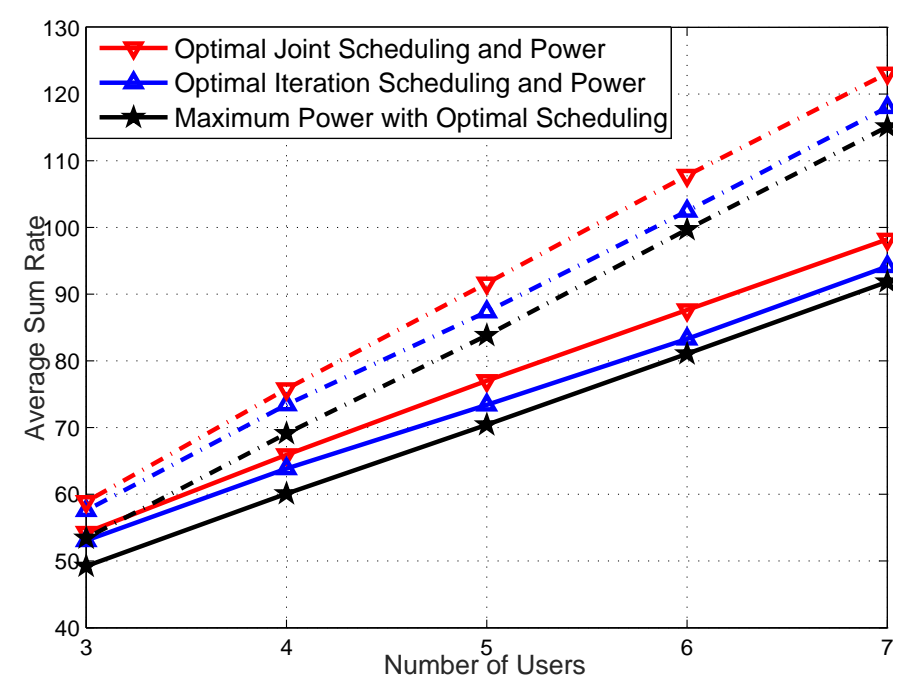

Fig. 5. Sum-rate in bps/Hz versus number of users $U$. Number of basestations is 3 , with 4 power-zones per BS's transmit frame, a maximum transmit power $P^{\max }=-42.60 \mathrm{dBm}$, and cell size $C=500$. The dashed lines illustrate the optimal solution to the maximum-weight clique problem while the solid ones are obtained by solving the problem using Algorithm 2.

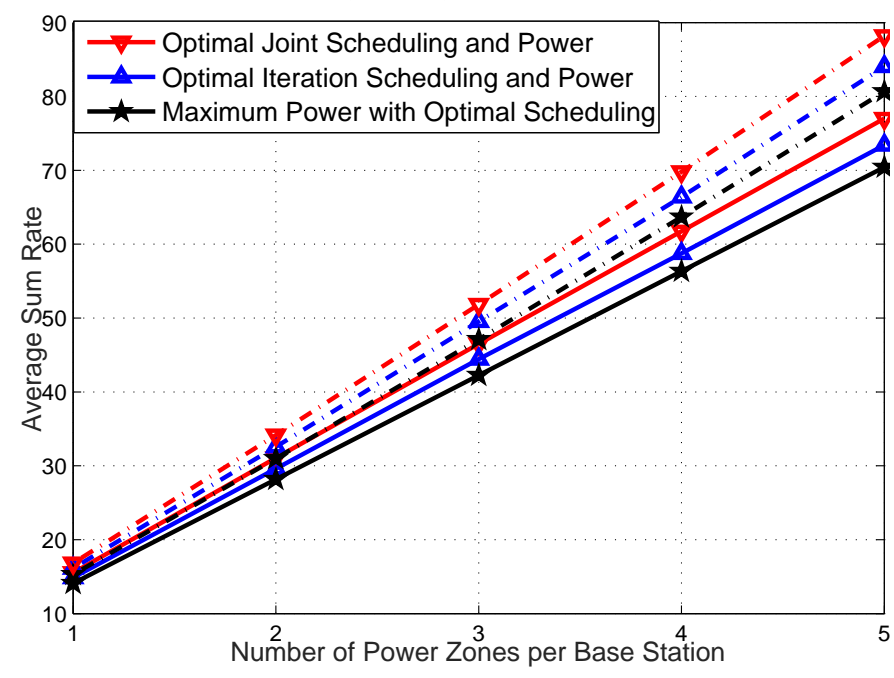

Fig. 6. Sum-rate in bps/Hz versus number of power-zones $Z$. Number of base-stations is 3 . Number of users is 5 . Maximum transmit power is $P^{\max }=$ $-42.60 \mathrm{dBm} / \mathrm{Hz}$, and cell size is $C=500$. The dashed lines illustrate the optimal solution to the maximum-weight clique problem while the solid ones are obtained by solving the problem using Algorithm 2 .

control outperforms iterative scheduling and power control scheme as the number of power-zones per frame increases, since the size of the search space becomes larger, which comes in the favour of our optimal algorithm.

Figure 7 and Figure 8 plot the sum-rate in $\mathrm{bps} / \mathrm{Hz}$ versus the maximum power $P^{\max }$, for a CRAN composed of 3 basestations, 5 power-zones and 5 users for a big cell network (cell size $C=500 \mathrm{~m}$ ) and a small cell network (cell size $C=100 \mathrm{~m}$ ), respectively. Both figures suggest that our joint optimization scheme outperforms all the other power allocation schemes particularity for large maximum power. The gain can be explained by the fact that as the maximum allowed power increases, the inter base-stations interference increases, which makes the role of joint scheduling and power control algorithm more vital. Similarly, when the cell size decreases, interference become larger. This clearly can be seen

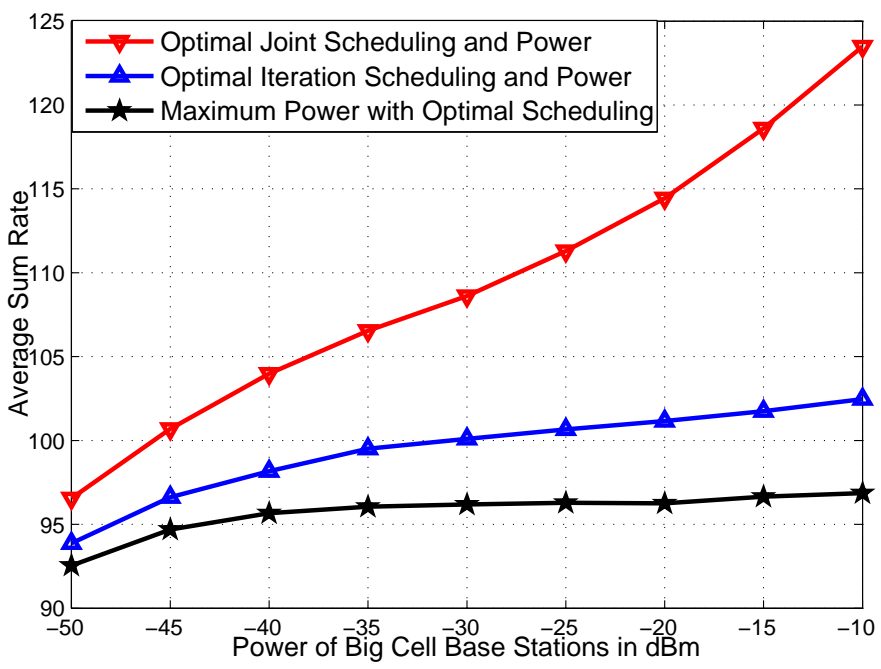

Fig. 7. Sum-rate in bps/Hz versus maximum allowed power $P^{\max }$. Number of base-stations is 3 , with 5 power-zones per BS's transmit frame and cell size $C=500 \mathrm{~m}$. Number of users is 10 .

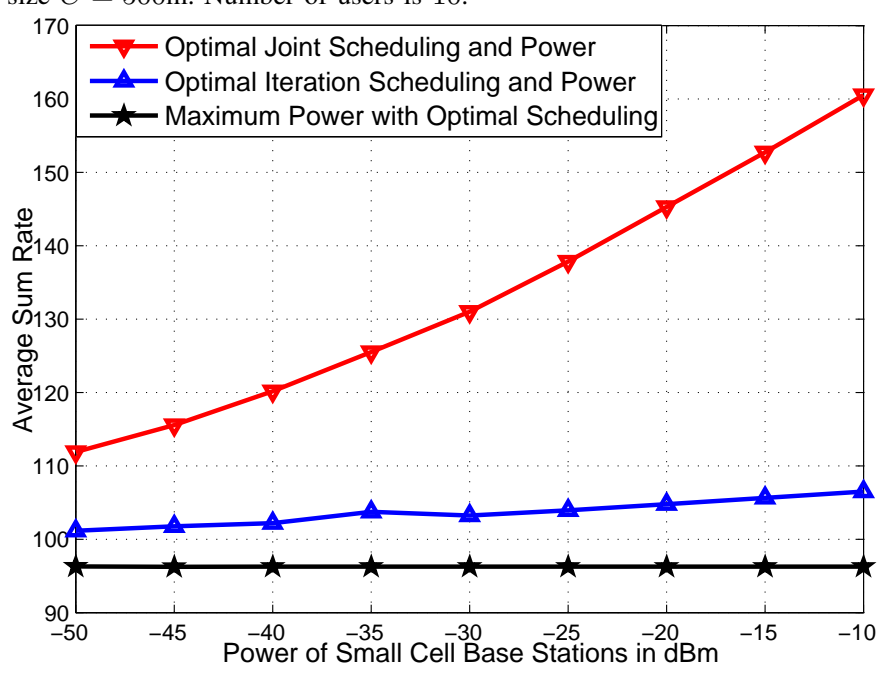

Fig. 8. Sum-rate in bps/Hz versus maximum allowed power $P^{\max }$. Number of base-stations is 3, with 5 power-zones per BS's transmit frame and cell size $C=100 \mathrm{~m}$. Number of users is 10 .

from Figure 8 in which the gain of our joint optimization scheme reaches up to $50 \%$ in a small cell scenario as compared to a gain of $20 \%$ in a big cell network Figure 7 .

Figure 9 plots the sum-rate in bps/Hz versus the cell size, for a CRAN composed of 3 base-stations, 5 power-zones, 5 users, and a maximum allowed power $P^{\max }=-26.98 \mathrm{dBm} / \mathrm{Hz}$. The joint scheduling and power optimization scheme presents a noticeable gain compared to the iterative scheduling and power optimization algorithm particularly at the high interference level. For a small cell network $(50 \leq C \leq 200 \mathrm{~m})$, our proposed solution achieves an appreciable performance improvement (between $45 \%$ for $C=50 \mathrm{~m}$ and $20 \%$ for $C=200 \mathrm{~m}$ ). For a big cell scenario, the proposed scheme achieves $10 \%$ performance improvement over the iterative algorithm and $15 \%$ improvement as compared to the maximum power allocation strategy.

Finally, to assess the performance of the proposed scheme in large CRAN, Figure 10 illustrates the sum-rate in bps $/ \mathrm{Hz}$ versus the number of users for a CRAN composed of 18 


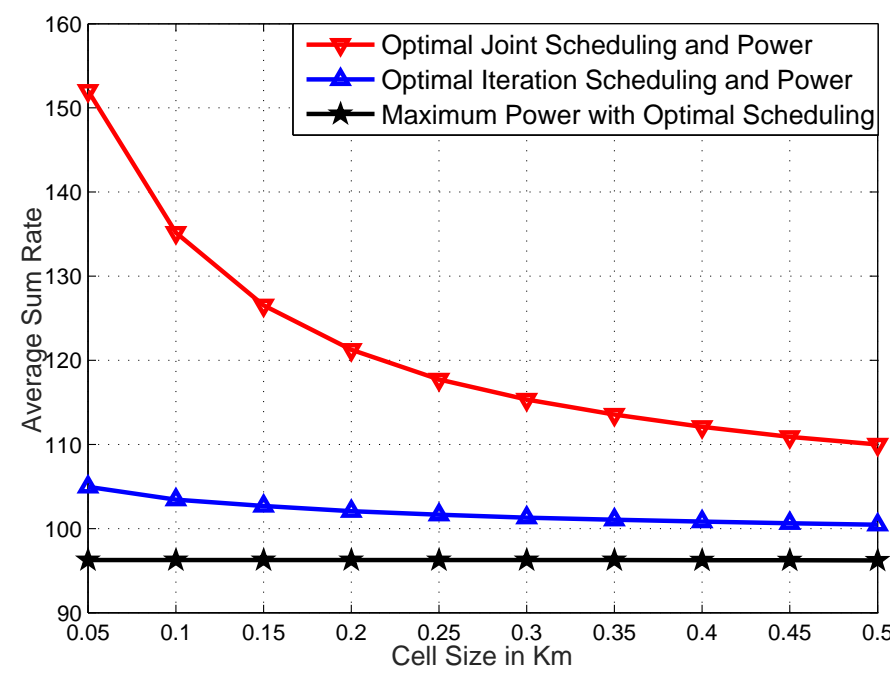

Fig. 9. Sum-rate in bps/Hz versus number of cell size $C$. Number of basestations is 3 , with 5 power-zones per BS's transmit frame, and a maximum power $P^{\max }=-26.98 \mathrm{dBm} / \mathrm{Hz}$. Number of users is 10 .

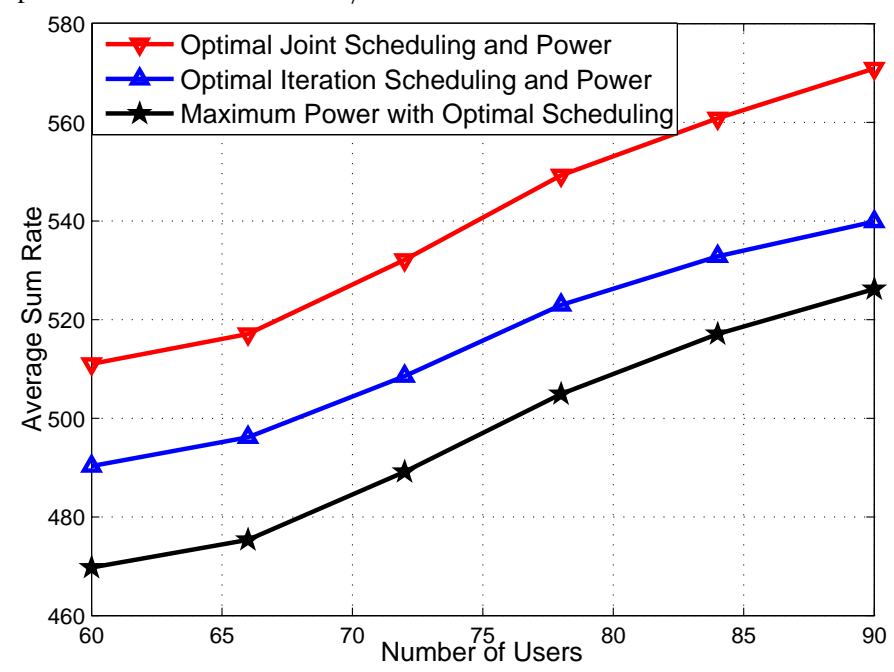

Fig. 10. Sum-rate in bps/Hz versus number of users $U$ for a large network. Number of base-stations is 18 , with 4 power-zones per BS's transmit frame, a maximum transmit power $P^{\max }=-42.60 \mathrm{dBm}$, and cell size $C=500$. The SINR gap is $1 \mathrm{dbm}$.

base-stations and 4 power-zones per frame. The figures shows that the proposed joint scheduling and power optimization scheme outperforms both the iterative and the maximum power approaches, especially as the number of users increases. This can be explained by the fact that, as the number of users increases, the scheduling opportunities with power adaptation increase, which results in a noticeable performance gain.

\section{CONCLUSION}

Optimization in cloud radio access networks is an emerging topic of interest of late. This paper considers the joint coordinated scheduling and power optimization problem in cloud-enabled networks. The paper solves the problem of maximizing the sum rate under the practical constraints that each user cannot be served by more than one base-station, but can be served by one or more power-zones within each basestation frame. A graph theoretical approach is used to solve the problem by introducing the joint scheduling and power control graph formed by several clusters, where every cluster is formed by a set of vertices, each representing the possible association of users, BSs, and PLs for one specific PZ. The problem is, then, formulated as a maximum-weight clique, in which the weight of each vertex is the sum of the benefits of the individual associations belonging to that vertex. Simulation results suggest that the proposed cross-layer scheme provides appreciable performance improvement as compared to other schemes from the literature, especially at high interference level.

\section{APPENDIX A PROOF OF LEMMA 1}

Let $\mathbf{S}=\left\{s_{1}, \cdots, s_{|\mathbf{S}|}\right\}$ be a schedule satisfying the second constraint $\mathrm{C} 2$. The mathematical formulation of this constraint is:

$$
\begin{aligned}
& \left(\varphi_{b}\left(s_{i}\right), \varphi_{z}\left(s_{i}\right)\right) \neq\left(\varphi_{b}\left(s_{j}\right), \varphi_{z}\left(s_{j}\right)\right), \forall 1 \leq i \neq j \leq|\mathbf{S}| \\
& \left|\bigcup_{i=1}^{|\mathbf{S}|}\left\{\left(\varphi_{b}\left(s_{i}\right), \varphi_{z}\left(s_{i}\right)\right)\right\}\right|=Z_{\mathrm{tot}} .
\end{aligned}
$$

Since all the $\left\{\varphi_{b}\left(s_{i}\right), \varphi_{z}\left(s_{i}\right)\right\}$ are disjoint, we can write:

$$
\begin{aligned}
Z_{\mathrm{tot}}=\left|\bigcup_{i=1}^{|\mathbf{S}|}\left\{\left(\varphi_{b}\left(s_{i}\right), \varphi_{z}\left(s_{i}\right)\right)\right\}\right| & =\sum_{i=1}^{|\mathbf{S}|}\left|\left\{\left(\varphi_{b}\left(s_{i}\right), \varphi_{z}\left(s_{i}\right)\right)\right\}\right| \\
& =\sum_{i=1}^{|\mathbf{S}|} 1=|\mathbf{S}| .
\end{aligned}
$$

Therefore, we can write the following:

$$
\begin{aligned}
& \left(\varphi_{b}(s), \varphi_{z}(s)\right) \neq\left(\varphi_{b}\left(s^{\prime}\right), \varphi_{z}\left(s^{\prime}\right)\right), \forall s \neq s^{\prime} \in \mathbf{S}, \\
& |\mathbf{S}|=Z_{\text {tot }} .
\end{aligned}
$$

If $\mathbf{S}$ further satisfies the first constraint $\mathbf{C} 1$, we can write:

$$
\varphi_{u}\left(s_{i}\right)=\varphi_{u}\left(s_{j}\right) \Rightarrow \varphi_{b}\left(s_{i}\right)=\varphi_{b}\left(s_{j}\right), \forall 1 \leq i \leq|\mathbf{S}| .
$$

The above condition can simply be rewritten as:

$$
\delta\left(\varphi_{u}\left(s_{i}\right)-\varphi_{u}\left(s_{j}\right)\right) \varphi_{b}\left(s_{i}\right)=\varphi_{b}\left(s_{j}\right) \delta\left(\varphi_{u}\left(s_{i}\right)-\varphi_{u}\left(s_{j}\right)\right) .
$$

By definition of the set of associations $\mathcal{A}$, all the PLs are included in the set $\mathcal{P}$. Therefore, all schedules $\mathbf{S} \in \mathcal{P}(\mathcal{A})$ verify $\mathrm{C} 3$. Finally, $\mathcal{F}$, defined as the schedules satisfying $\mathrm{C} 1$, $\mathrm{C} 2$, and $\mathrm{C} 3$, can be written as:

$$
\begin{aligned}
& \mathcal{F}=\left\{\mathbf{S} \in \mathcal{P}(\mathcal{A}) \text { such that } \forall s \neq s^{\prime} \in \mathbf{S}\right. \\
& \delta\left(\varphi_{u}(s)-\varphi_{u}\left(s^{\prime}\right)\right) \varphi_{b}(s)=\varphi_{b}\left(s^{\prime}\right) \delta\left(\varphi_{u}(s)-\varphi_{u}\left(s^{\prime}\right)\right), \\
& \left(\varphi_{b}(s), \varphi_{z}(s)\right) \neq\left(\varphi_{b}\left(s^{\prime}\right), \varphi_{z}\left(s^{\prime}\right)\right), \\
& \left.|\mathbf{S}|=Z_{\text {tot }}\right\} .
\end{aligned}
$$

\section{APPENDIX B \\ Proof of TheOREM 1}

To prove this theorem, we first show that for any feasible schedule, there exists a unique clique in the joint scheduling and power control graph that represents the schedule. We then prove that for any clique of size $Z$, there exist a unique schedule that is feasible. Hence, we prove that there is a one to one mapping between the set of clique of size $Z$ and the set of the feasible schedule. To conclude the proof we show that the weight of the clique is the objective function (3) to maximize. 
Let $\mathbf{S}=\left\{s_{1}, \cdots, s_{Z_{\text {tot }}}\right\}$ be a feasible schedule (i.e. $\mathbf{S} \in \mathcal{F}$ ). This schedule contains $Z_{\text {tot }}=Z \times B$ associations. To prove that $\mathbf{S}$ can be represented by a clique in the joint scheduling and power control graph, we prove that $\mathbf{S}$ can be decomposed into $Z$ subset of associations each representing a vertex in a local power control graph. Finally, we prove that the vertices are connected in the joint scheduling and power control graph.

Let $S_{z}=\left\{s \in \mathbf{S} \mid \varphi_{z}(s)=z\right\}, z \in \mathcal{Z}$ be the set of associations which PZs is indexed by $z$. From the feasibility conditions of a schedule (4b), we have $\left|S_{z}\right|=B$. The schedule $\mathbf{S}$ is feasible and thus satisfy (4a), (4b) and (4c). Therefore, $S_{z}$ satisfy (4b) and (4c) which concludes that $S_{z} \in \mathcal{F}^{z}$. This proves that $S_{z}$ is represented by a vertices in the local power control graph $\mathcal{G}^{z}$.

We now prove that all the vertices $S_{z}, z \in \mathcal{Z}$ are connected in the joint scheduling and power control graph. given that the schedule satisfies (4a), we clearly see, from the connectivity condition (8) in the joint scheduling and power control graph, that all the vertices are pairwise connected. Therefore, $\mathbf{C}=$ $\left\{S_{1}, \cdots, S_{Z}\right\}$ is a clique of size $Z$ in the joint scheduling and power control graph. The decomposition of $\mathbf{S}$ into clusters $S_{z}, z \in \mathcal{Z}$ is unique up to index permutation. Hence, the clique representing the schedule is unique. We now prove the converse.

Define $\mathcal{C}$ as the set of cliques of degree $Z$ in the joint scheduling and power control graph $\mathcal{G}=\bigcup_{z \in \mathcal{Z}} \mathcal{G}^{z}$ where $\mathcal{G}^{z}$ is the local power control graph indexed by the PZ $z$. Let $\mathbf{C}=\left\{v_{1}, \cdots, v_{Z}\right\} \in \mathcal{C}$ be a clique. Note that by definition of the local power control graph, each vertex $v_{z} \in \mathcal{G}^{z}$ represents $B$ associations. Therefore, $\mathbf{C}$ satisfy condition (4c) since $|\mathbf{C}|=\sum_{z \in \mathcal{Z}}\left|v_{z}\right|=Z \times B=Z_{\text {tot }}$. By construction of the vertices $v_{z} \in \mathcal{G}^{z}$, we have $v_{z} \in \mathcal{F}^{z}$. Therefore, vertices in the clusters satisfy (4b). Each cluster represents a different $\mathrm{PZ}$, then by extension $\mathrm{C}$ satisfy (4b). The following lemma characterize the vertices included in a clique $\mathbf{C}$ of size $Z$ (i.e. $\mathbf{C} \in \mathcal{C})$.

Lemma 3. Let $\mathbf{C}=\left\{v_{1}, \cdots, v_{Z}\right\} \in \mathcal{C}$ be a clique of size $Z$ in the joint scheduling and power control graph. There exist a one to one mapping between vertices $v_{z}, z \in \mathcal{Z}$ and the clusters $\mathcal{G}^{z}, z \in \mathcal{Z}$. In other words, two distinct vertices belongs to two different clusters.

\section{Proof: The proof can be found in Appendix F}

Using result of Lemma 3 and the connectivity condition between two distinct clusters (8), clearly, $\mathbf{C}$ satisfy (4a). As a conclusion, $\mathbf{C}$ represents a feasible schedule. The uniqueness of the schedule follows from the unique nature of vertices in each cluster. Therefore, there exist a one to one mapping between the set of feasible schedules and the set of cliques in the graph. We now prove that the weight of the clique is the objective function in (3) to be maximized.

Let $\mathbf{C}=\left\{v_{1}, \cdots, v_{Z}\right\}=\left\{s_{1}, \cdots, s_{Z_{\text {tot }}}\right\}=\mathbf{S}, v_{z} \in \mathcal{G}^{z}$. Let $v^{z}$ be associated with the schedule $\mathbf{S}^{z}=\left\{s_{1}^{z}, \cdots, s_{B}^{z}\right\} \in$
$\mathcal{F}^{z}$. The weight of the clique can be expressed as:

$$
\begin{aligned}
w(\mathbf{C}) & =\sum_{z=1}^{Z} w\left(v_{z}\right) \\
& =\sum_{z=1}^{Z} \sum_{i=1}^{B} a\left(s_{i}^{z}\right) \mathfrak{g}\left(s_{i}^{z}, \mathbf{S}\right)=\sum_{i=1}^{Z \times B} a\left(s_{i}\right) \mathfrak{g}\left(s_{i}, \mathbf{S}\right) \\
& =\sum_{i=1}^{Z_{\text {tot }}} a\left(s_{i}\right) \mathfrak{g}\left(s_{i}, \mathbf{S}\right)=w(\mathbf{S})
\end{aligned}
$$

Therefore, the optimal solution of the optimization problem (3) can be written as:

$$
\begin{aligned}
\mathbf{S}^{*} & =\arg \max _{\mathbf{S} \in \mathcal{F}} \sum_{s \in \mathbf{S}} a(s) \mathfrak{g}(s, \mathbf{S}) \\
& =\arg \max _{\mathbf{C} \in \mathcal{C}} \sum_{v \in \mathbf{C}} w(v),
\end{aligned}
$$

where $\mathbf{C}=\left\{v_{1}, \cdots, v_{Z}\right\} \in \mathcal{C}$ is a clique in the joint scheduling and power control graph, $\mathcal{C}$ is the set of all possible cliques with degree $Z$, and $w(v)$ is the weight of each corresponding vertex. In other words, the optimal solution of the optimization problem (3) is the maximum-weight clique of degree $Z$ where the weight of each vertex $v^{z} \in \mathcal{V}$ associated with $\mathbf{S}^{z}=\left\{s_{1}, \cdots, s_{B}\right\} \in \tilde{\mathcal{F}}^{z}$ is:

$$
w\left(v^{z}\right)=\sum_{i=1}^{B} a\left(s_{i}^{z}\right) \mathfrak{g}\left(s_{i}^{z}, \mathbf{S}^{z}\right)
$$

\section{APPENDIX C \\ PROOF OF THEOREM 2}

First note that given the optimal PLs, the optimal solution can be achieved using Theorem 1. In other words, if $\mathbf{S}^{*}=\left\{s_{1}^{*}, \cdots, s_{Z}^{*}\right\}$ is the optimal solution to the scheduling problem with the optimal power control and if $\left.\left\{\varphi_{p}\left(s_{1}\right), \cdots, \varphi_{p}\left(s_{Z}\right)\right)\right\} \subseteq \mathcal{P}$, then the solution is given by the maximum-weight clique in the joint scheduling and power control graph. Therefore, to prove this theorem, we first show that for a particular PLs, only a subset of vertices in the clusters are needed to achieve the optimal solution. We, then prove that the optimal PLs are generated by Algorithm 1.

Consider a cluster $\mathcal{G}^{z}$ and define the equivalence relationship $\mathbf{R}$ between two vertices $v^{z}=\left\{v_{1}^{z}, \cdots, v_{B}^{z}\right\}$ and $u^{z}=\left\{u_{1}^{z}, \cdots, u_{B}^{z}\right\}$ in the same cluster as follows:

$$
\begin{gathered}
\left\{\varphi_{u}\left(v_{i}^{z}\right), \varphi_{b}\left(v_{i}^{z}\right), \varphi_{z}\left(v_{i}^{z}\right)\right\}=\left\{\varphi_{u}\left(u_{i}^{z}\right), \varphi_{b}\left(u_{i}^{z}\right), \varphi_{z}\left(u_{i}^{z}\right)\right\} \\
, 1 \leq i \leq B .
\end{gathered}
$$

In other words, two vertices are associated with $\mathbf{R}$ if they represent the same associations up to a PL change. Clearly, $\mathbf{R}$ is reflexive $\left(v^{z} \mathbf{R} v^{z}\right)$, symmetric $\left(v^{z} \mathbf{R} u^{z} \Rightarrow u^{z} \mathbf{R} v^{z}\right)$ and transitive $\left(v^{z} \mathbf{R} u^{z}, u^{z} \mathbf{R} w^{z} \Rightarrow v^{z} \mathbf{R} w^{z}\right)$. Hence, it is an equivalence relationship. Let $\mathbf{R}\left(v^{z}\right)$ be the equivalence class of $v^{z}$. The following Lemma characterizes the vertices of the maximum-weight clique:

Lemma 4. For each equivalence class $\mathbf{R}\left(v^{z}\right)$, only the vertex with the highest weight is needed to achieve the optimal solution of the scheduling problem (2).

Proof: The proof can be found in Appendix F

To conclude the proof, we show that, for each cluster and each equivalence class in it, Algorithm 1 produces the vertex 
with the highest weight. Assume that $\mathbf{S}^{*}=\left\{s_{1}^{*}, \cdots, s_{Z}^{*}\right\}$ is the optimal solution for the scheduling problem with optimal power control. Assume that $\exists j$ such that $s_{j}^{*}$ is not generated by the algorithm. Let $\mathbf{R}\left(s_{j}^{*}\right)$ be the equivalence class of $s_{j}^{*}$. From Lemma 4 , we have $w\left(s_{j}^{*}\right) \geq w(v), \forall v \in \mathbf{R}\left(s_{j}^{*}\right)$. The vertex generated $v^{*}$ by the algorithm is the optimal solution to (14), thus $w\left(v^{*}\right) \geq w(v), \forall v \in \mathbf{R}\left(s_{j}^{*}\right)$. Therefore, we obtain $w\left(v^{*}\right) \geq w\left(s_{j}^{*}\right)$ but $\mathbf{S}^{*}$ is the optimal solution then $w\left(s_{j}^{*}\right)=$ $w\left(v^{*}\right)$. From Lemma 4, we know that all vertices in the same clusters have the same connections, which conclude that the algorithm produces the same solution (if $s_{j}=v^{*}$ ) or a solution with the same weight $\left(w\left(s_{j}^{*}\right)=w\left(v^{*}\right)\right.$ and $\left.s_{j}^{*} \neq v^{*}\right)$ in which case the optimal solution is not unique. As a conclusion, the optimal coordinated scheduling solution with optimal power control (2) is achieved using Algorithm 1.

\section{APPENDIX D \\ PROOF OF LEMMA 2}

The complexity of reaching the optimal solution using Algorithm 1 can be decomposed in the complexity of generating the joint scheduling and power control graph and the complexity of solving the maximum-weight clique in that graph. We first look at the complexity of producing a single cluster. From Lemma 4, for an arbitrary cluster, the number of vertices is the number of distinct equivalence classes the cluster contains. For cluster $z$, this figure is equal to the total number of the feasible schedules is $\left|\tilde{\mathcal{F}}^{z}\right|$. Since the number of association each vertex contain is $B$, which is equal to the number of BSs, then the total number of possible vertices is the number of permutations of the users among these BSs. This number can be computed as ${ }^{U} P_{B}$. For each vertex, the optimization problem (14) is run once to calculate the optimal power level. Hence, the complexity of creating a cluster is ${ }^{U} P_{B} Z c(B)$. The whole graph contains ${ }^{U} P_{B} Z$ which conclude that the total complexity for achieving the optimal solution is ${ }^{U} P_{B} Z c(B)+f\left({ }^{U} P_{B} Z\right)$.

\section{APPENDIX E ProOF OF THEOREM 3}

To prove Theorem 3, we show that there is a one to one map between $\mathcal{F}$ (i.e., the set of feasible schedules) and $\mathcal{C}$ (i.e., the set of cliques of degree $Z_{\text {tot }}$ in the scheduling graph $\mathcal{G}(\mathcal{V}, \mathcal{E})$ ). We first prove that $\forall \mathbf{C} \in \mathcal{C}, \mathbf{C}$ satisfies the constraints (4a), (4b) and (4c). Then, we prove the converse: i.e. for each element in $\mathbf{S} \in \mathcal{F}$, there exists an associated clique $\mathbf{C} \in \mathcal{C}$. To conclude the proof, we show that the weight of the clique is equivalent to the merit function of the optimization problem defined in (12).

Let $\mathbf{C}=\left\{v_{1}, \cdots, v_{|\mathbf{C}|}\right\}$ be a clique in the scheduling graph $\mathcal{G}(\mathcal{V}, \mathcal{E})$ (i.e., $\mathbf{C} \in \mathcal{C}$ ). Since $\mathbf{C}$ is a clique in $\mathcal{G}$, there exists an edge in $\mathcal{E}$ for every pair of vertices in $\mathcal{V}$. From the first condition $\mathrm{C} 1$ of creating an edge between two vertices, we have:

$$
\begin{aligned}
\varphi_{u}\left(v_{i}\right)=\varphi_{u}\left(v_{j}\right) \text { and } \varphi_{b}\left(v_{i}\right) & =\varphi_{b}\left(v_{j}\right), \forall 1 \leq i \neq j \leq|\mathbf{C}|, \\
\delta\left(\varphi_{u}\left(v_{i}\right)-\varphi_{u}\left(v_{j}\right)\right) \varphi_{b}\left(v_{i}\right) & =\varphi_{b}\left(v_{j}\right) \delta\left(\varphi_{u}\left(v_{i}\right)-\varphi_{u}\left(v_{j}\right)\right) .
\end{aligned}
$$

Hence, the clique satisfies (4a). In a similar way, the second condition $\mathrm{C} 2$ of the connectivity gives the following:

$$
\left(\varphi_{b}\left(v_{i}\right), \varphi_{z}\left(v_{i}\right)\right) \neq\left(\varphi_{b}\left(v_{j}\right), \varphi_{z}\left(v_{j}\right)\right), \forall 1 \leq i \neq j \leq|\mathbf{C}| .
$$

Therefore, for any $\mathbf{C}=\left\{v_{1}, \cdots, v_{|\mathbf{C}|}\right\} \in \mathcal{C}$, we can construct a schedule $\mathbf{S}$, such that $\mathbf{S}=\left\{s_{1}, \cdots, s_{|\mathbf{C}|}\right\} \in \mathcal{P}(\mathcal{A})$, where $v_{i}$ is the vertex associated with each association $s_{i}, \forall 1 \leq i \leq$ $|\mathbf{C}|$, and where $\mathbf{S}$ satisfies the following constraints:

$$
\begin{aligned}
& \mathbf{S} \in \mathcal{P}(\mathcal{A}), \text { and } \forall s \neq s^{\prime} \in \mathbf{S} \\
& \delta\left(\varphi_{u}(s)-\varphi_{u}\left(s^{\prime}\right)\right) \varphi_{b}(s)=\varphi_{b}\left(s^{\prime}\right) \delta\left(\varphi_{u}(s)-\varphi_{u}\left(s^{\prime}\right)\right), \\
& \left(\varphi_{b}(s), \varphi_{z}(s)\right) \neq\left(\varphi_{b}\left(s^{\prime}\right), \varphi_{z}\left(s^{\prime}\right)\right), \\
& |\mathbf{S}|=|\mathcal{C}|=Z_{\text {tot }}
\end{aligned}
$$

The conclusion in E. 1 shows that $\mathbf{S} \in \mathcal{F}$.

In a similar manner, let $\mathbf{S}=\left\{s_{1}, \cdots, s_{|\mathbf{S}|}\right\} \in \mathcal{F}$, and let $\mathbf{C}=\left\{v_{1}, \cdots, v_{|\mathbf{C}|}\right\}$ where $v_{i}$ is the vertex associated with $s_{i}, \forall 1 \leq i \leq|\mathbf{C}|$. Due to conditions (4a) and (4b), each pair of vertices are connected; thus, $\mathbf{C}$ is a clique. Further, (4c) guarantees that the size of the clique is $Z_{\mathrm{tot}}$. This concludes the converse, i.e., $\mathbf{C} \in \mathcal{C}$.

Moreover, the weight of the clique $\mathbf{C} \in \mathcal{C}$ is simply given by:

$$
w(\mathbf{C})=\sum_{i=1}^{|\mathbf{C}|} w\left(v_{i}\right)=\sum_{i=1}^{|\mathbf{C}|} r\left(s_{i}\right),
$$

where $s_{i}$ is the association corresponding to vertex $v_{i}$. This implies that the weight of the clique is equivalent to the merit function of the optimization problem defined in (12). Hence, the optimal scheduling is given by the maximum-weight clique of degree $Z_{\text {tot }}$ in the scheduling graph.

\section{APPENDIX F \\ AUXILIARY LEMMAS}

\section{A. Proof of Lemma 3}

To prove this Lemma, we first show that for a given schedule to satisfy condition (4b), the local power control graph for any power-zone should not contain any connection.

Assume there exists a connection between two distinct vertices $v_{i}$ and $v_{j}$ belonging to the same cluster $\mathcal{G}^{z}$. By construction of the cluster $\mathcal{G}^{z}$ each vertex represents $B$ associations satisfying condition (4b) and (4c). These $B$ associations have the same PZs indexed by $z$ and satisfy the constraints. Given that exactly $B \mathrm{PZs}$ indexed by $z$ across the entire network that need to be scheduled, all these $B$ PZs indexed by $z$ have been associated with one user. Vertices $v_{i}$ and $v_{j}$ are distinct vertices, there exist at least one association that is different and, therefore, there exist a BS whose $z$ th $\mathrm{PZ}$ is associated with various users which violate condition (4b).

Let $\mathbf{C}=\left\{v_{1}, \cdots, v_{Z}\right\} \in \mathcal{C}$ be a clique that represents a feasible schedule. Assume that two distinct vertices $v_{i}$ and $v_{j}, 1 \leq i \neq j \leq Z$ belong to the same cluster. Since these vertices are part of the clique $\mathbf{C}$, then they are connected by an edge. But as shown previously, such connection does not exist otherwise the schedule is not feasible. Therefore, two distinct vertices belongs to two different clusters. The number of vertices in the clique being equal to the number of clusters, there exists a one to one mapping between the vertices of $\mathbf{C} \in \mathcal{C}$ and the clusters $\mathcal{G}^{z}, z \in \mathcal{Z}$. 


\section{B. Proof of Lemma 4}

Let $v^{z}$ be a vertex in the cluster $\mathcal{G}^{z}$ and let $\mathbf{R}\left(v^{z}\right)$ be its equivalence class. To show that only the vertex with the highest weight is needed to achieve the optimal solution, we will show that all vertices belonging to $\mathbf{R}\left(v^{z}\right)$ are connected to the same vertices. Let $v^{z}$ and $u^{z}$ be two arbitrary vertices in $\mathbf{R}\left(v^{z}\right)$. From Lemma 3, vertices in the same cluster are not connected to each other. Hence, they have the same connections with vertices in the cluster $\mathcal{G}^{z}$. We now prove that they have the same connections with vertices in cluster $\mathcal{G}^{m}, m \in \mathcal{Z}, m \neq z$. Assume $v^{z}$, associated with the schedule $\mathbf{S}^{z}$, is connected to $v^{m}$, associated with the schedule $\mathbf{S}^{m}$, then from the connectivity condition between two different cluster (8), we have:

$$
\begin{aligned}
& \delta\left(\varphi_{u}\left(s^{z}\right)-\varphi_{u}\left(s^{m}\right)\right) \delta\left(\varphi_{b}\left(s^{z}\right)-\varphi_{b}\left(s^{m}\right)\right) \\
& =\delta\left(\varphi_{u}\left(s^{z}\right)-\varphi_{u}\left(s^{m}\right)\right), \forall\left(s^{z}, s^{m}\right) \in \mathbf{S}^{z} \times \mathbf{S}^{m}
\end{aligned}
$$

Since $v^{z}$ and $u^{z}$ are linked with the equivalence relationship $\mathbf{R}$, then

$$
\begin{gathered}
\left\{\varphi_{u}\left(v_{i}^{z}\right), \varphi_{b}\left(v_{i}^{z}\right), \varphi_{z}\left(v_{i}^{z}\right)\right\}=\left\{\varphi_{u}\left(u_{i}^{z}\right), \varphi_{b}\left(u_{i}^{z}\right), \varphi_{z}\left(u_{i}^{z}\right)\right\} \\
, 1 \leq i \leq B .
\end{gathered}
$$

Substituting (F.2) in (F.1), we obtain:

$$
\begin{aligned}
& \delta\left(\varphi_{u}\left(s^{z}\right)-\varphi_{u}\left(t^{z}\right)\right) \delta\left(\varphi_{b}\left(s^{z}\right)-\varphi_{b}\left(t^{z}\right)\right) \\
& =\delta\left(\varphi_{u}\left(s^{z}\right)-\varphi_{u}\left(t^{z}\right)\right), \forall\left(s^{z}, t^{z}\right) \in \mathbf{S}^{z} \times \mathbf{T}^{z}
\end{aligned}
$$

where $u^{z} \in \mathbf{R}\left(v^{z}\right)$ associated with the schedule $\mathbf{T}^{z}$. the above equality concludes that $u^{z}$ and $v^{m}$ are connected. Since $v^{z}$ and $u^{z}$ play symmetric roles, then the vertices have the same connections with vertices in cluster $\mathcal{G}^{m}, m \in \mathcal{Z}, m \neq z$. Finally, we conclude that the two vertices have the same connection in the whole graph.

Assume $\mathbf{S}^{*}=\left\{s_{1}^{*}, \cdots, s_{Z}^{*}\right\}$ is the optimal solution to the scheduling problem and assume that $\exists j$ such that $s_{j}^{*}$ is not the vertex with the maximum weight in $\mathbf{R}\left(s_{j}^{*}\right)$. Let $s_{j}^{\dagger}$ be the vertex with highest weight in $\mathbf{R}\left(s_{j}^{*}\right)$. Since $s_{j}^{*}$ and $s_{j}^{\dagger}$ have the same connections then $s_{j}^{\dagger}$ is connected to all $s_{i}^{*}, 1 \leq i \neq j \leq$ $Z$. Therefore, $\mathbf{S}^{\dagger}=\left(\mathbf{S}^{*} \backslash s_{j}^{*}\right) \cup s_{j}^{\dagger}$ is also a clique of size $Z$ and with a higher weight than $\mathbf{S}^{*}$ which is in contradiction that $\mathbf{S}^{*}$ is the optimal solution and thus the maximum-weight clique of size $Z$. As a conclusion, only the vertices with the highest weight in their equivalence class are needed to reach the optimal solution.

\section{REFERENCES}

[1] A. Douik, H. Dahrouj, T. Y. Al-Naffouri, and M.-S. Alouini, "Coordinated scheduling for the downlink of cloud radio-access networks," Proc. of IEEE International Conference on Communications (ICC' 2015), London, UK, avaialble Arxiv e-prints, vol. abs/1004.1379, 2014.

[2] J. Andrews, S. Buzzi, W. Choi, S. Hanly, A. Lozano, A. Soong, and J. Zhang, "What will 5G be?" IEEE Journal on Selected Areas in Communications, vol. 32, no. 6, pp. 1065-1082, June 2014.

[3] S.-H. Park, O. Simeone, O. Sahin, and S. Shamai, "Joint precoding and multivariate backhaul compression for the downlink of cloud radio access networks," IEEE Transactions on Signal Processing, vol. 61, no. 22, pp. 5646-5658, Nov 2013.

[4] B. Dai and W. Yu, "Sparse beamforming for limited-backhaul network mimo system via reweighted power minimization," in Proc. of IEEE Global Telecommunications Conference (GLOBECOM' 2013), Atlanta, GA, USA, Dec 2013, pp. 1962-1967.

[5] Y. Shi, J. Zhang, and K. Letaief, "Group sparse beamforming for green cloud-RAN," IEEE Transactions on Wireless Communications, vol. 13, no. 5, pp. 2809-2823, May 2014.
[6] W. Yu, T. Kwon, and C. Shin, "Joint scheduling and dynamic power spectrum optimization for wireless multicell networks," in Proc. of 2010 44th Annual Conference on Information Sciences and Systems (CISS' 2010), Princeton, New Jersey, USA, March 2010, pp. 1-6.

[7] — , "Multicell coordination via joint scheduling, beamforming, and power spectrum adaptation," IEEE Transactions on Wireless Communications, vol. 12, no. 7, pp. 1-14, July 2013.

[8] A. Stolyar and H. Viswanathan, "Self-organizing dynamic fractional frequency reuse for best-effort traffic through distributed inter-cell coordination," in Proc. of 28th IEEE Conference on Computer Communications (INFOCOM' 2009), Rio de Janeiro, Brazil, April 2009, pp. 1287-1295.

[9] H. Dahrouj, W. Yu, T. Tang, J. Chow, and R. Selea, "Coordinated scheduling for wireless backhaul networks with soft frequency reuse," in Proc. of the 21st Europea Signal Processing Conference (EUSIPCO' 2013), Marrakech, Morocco, Sept 2013, pp. 1-5.

[10] D. P. Bertsekas, "The auction algorithm: A distributed relaxation method for the assignment problem," Annals of Operations Research, vol. 14, pp. 105-123, 1988 .

[11] S. Kiani and D. Gesbert, "Optimal and distributed scheduling for multicell capacity maximization," IEEE Transactions on Wireless Communications, vol. 7, no. 1, pp. 288-297, Jan 2008.

[12] R. Bendlin, Y.-F. Huang, M. Ivrlac, and J. Nossek, "Fast distributed multi-cell scheduling with delayed limited-capacity backhaul links," in Proc. of IEEE International Conference on Communications (ICC' 2009), Dresden, Germany, June 2009, pp. 1-5.

[13] W. Choi and J. Andrews, "The capacity gain from intercell scheduling in multi-antenna systems," IEEE Transactions on Wireless Communications, vol. 7, no. 2, pp. 714-725, February 2008.

[14] L. Jiang, S. Parekh, and J. Walrand, "Base station association game in multi-cell wireless networks (special paper)," in Proc of IEEE Wireless Communications and Networking Conference (WCNC'2008), Las Vegas, Nevada, USA, March 2008, pp. 1616-1621.

[15] R. Sun, M. Hong, and Z.-Q. Luo, "Optimal joint base station assignment and power allocation in a cellular network," in Proc of IEEE 13th International Workshop on Signal Processing Advances in Wireless Communications (SPAWC' 2012), Cesme, Turkey, June 2012, pp. 234 238.

[16] J.-W. Lee, R. Mazumdar, and N. Shroff, "Joint resource allocation and base-station assignment for the downlink in CDMA networks," IEEE/ACM Transactions on Networking, vol. 14, no. 1, pp. 1-14, Feb 2006.

[17] M. Rahman and H. Yanikomeroglu, "Enhancing cell-edge performance: a downlink dynamic interference avoidance scheme with inter-cell coordination," IEEE Transactions on Wireless Communications, vol. 9, no. 4, pp. 1414-1425, April 2010.

[18] D. Gesbert, S. Kiani, A. Gjendemsjo, and G. ien, "Adaptation, coordination, and distributed resource allocation in interference-limited wireless networks," Proceedings of the IEEE, vol. 95, no. 12, pp. 2393-2409, Dec 2007.

[19] J. Huang, V. Subramanian, R. Agrawal, and R. Berry, "Downlink scheduling and resource allocation for OFDM systems," IEEE Transactions on Wireless Communications, vol. 8, no. 1, pp. 288-296, Jan 2009.

[20] _ , "Joint scheduling and resource allocation in uplink OFDM systems for broadband wireless access networks," IEEE Journal on Selected Areas in Communications, vol. 27, no. 2, pp. 226-234, February 2009.

[21] L. Venturino, N. Prasad, and X. Wang, "Coordinated scheduling and power allocation in downlink multicell OFDMA networks," IEEE Transactions on Vehicular Technology, vol. 58, no. 6, pp. 2835-2848, July 2009.

[22] B. Rengarajan, A. Stolyar, and H. Viswanathan, "Self-organizing dynamic fractional frequency reuse on the uplink of OFDMA systems," in Proc. of 2010 44th Annual Conference on Information Sciences and Systems (CISS' 2010), Princeton, New Jersey, USA, March 2010, pp. $1-6$.

[23] L. Yu, E. Karipidis, and E. Larsson, "Coordinated scheduling and beamforming for multicell spectrum sharing networks using branch and bound," in Proc. of the 20th European Signal Processing Conference (EUSIPCO' 2012), Bucharest, Romania, Aug 2012, pp. 819-823.

[24] P. R. J. Ostergard, "A fast algorithm for the maximum clique problem," Discrete Appl. Math, vol. 120, pp. 197-207.

[25] K. Yamaguchi and S. Masuda, "A new exact algorithm for the maximumweight clique problem," in Proc. Of the 23rd International Technical Conference on Circuits/Systems, Computers and Communications (ITCCSCC' 2008), Yamaguchi, Japan. 
[26] L. P. Qian, Y. Zhang, and J. Huang, "Mapel: Achieving global optimality for a non-convex wireless power control problem," IEEE Transactions on Wireless Communications, vol. 8, no. 3, pp. 1553-1563, March 2009.

[27] H. Dahrouj, W. Yu, and T. Tang, "Power spectrum optimization for interference mitigation via iterative function evaluation," EURASIP Journal on Wireless Communications and Networking, vol. 2012, no. 1, p. 244, 2012.

[28] S. Stanczak, M. Wiczanowski, and H. Boche, Fundamentals of resource allocation in wireless networks: theory and algorithms. Springer, 2009, vol. 3.

[29] H. Boche and M. Schubert, "A superlinearly and globally convergent algorithm for power control and resource allocation with general interference functions," Networking, IEEE/ACM Transactions on, vol. 16, no. 2, pp. 383-395, April 2008.

[30] S. Christensen, R. Agarwal, E. de Carvalho, and J. Cioffi, "Weighted sum-rate maximization using weighted MMSE for MIMO-BC beamforming design," in Proc. of IEEE International Conference on Communications (ICC' 2009), Dresden, Germany, June 2009, pp. 1-6.

[31] N. Bourgeois, B. Escoffier, V. T. Paschos, and J. M. M. van Rooij, "A bottom-up method and fast algorithms for max independent set," in Proc. of the 12th Scandinavian Conference on Algorithm Theory (SWAT" 2010), Bergen, Norway.

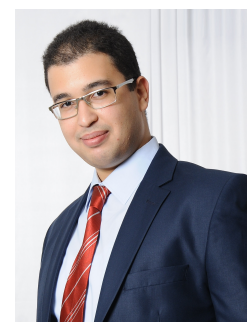

Ahmed Douik (S'13) received the Eng. degree in electronic and communication engineering (with first class honors) from the Ecole Polytechnique de Tunisie, Tunisia, in 2013, the M.S. degree in electrical engineering from King Abdullah University of Science and Technology, Thuwal, Saudi Arabia, in 2015. He is now pursuing his Ph.D. at the California Institute of Technology, Pasadena, CA, USA. His research interests include cloud-radio access networks, network coding, single and multi-hop transmissions, and cooperation communication.

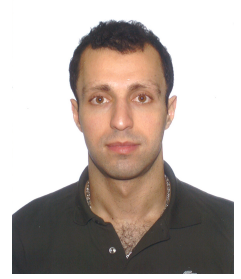

Hayssam Dahrouj (S'02, M'11, SM'15) received his B.E. degree (with high distinction) in computer and communications engineering from the American University of Beirut (AUB), Lebanon, in 2005, and his $\mathrm{Ph} . \mathrm{D}$. degree in electrical and computer engineering from the University of Toronto (UofT), Canada, in 2010. In May 2015, he joined the Department of Electrical and Computer Engineering at Effat University as an assistant professor, and also became a visiting scholar at King Abdullah University of Science and Technology (KAUST). Between April 2014 and May 2015, he was with the Computer, Electrical and Mathematical Sciences and Engineering group at KAUST as a research associate. Prior to joining KAUST, he was an industrial postdoctoral fellow at UofT, in collaboration with BLiNQ Networks Inc., Kanata, Canada, where he worked on developing practical solutions for the design of non-line-of sight wireless backhaul networks. His contributions to the field led to five patents. During his doctoral studies at UofT, he pioneered the idea of coordinated beamforming as a means of minimizing intercell interference across multiple base stations. The journal paper on this subject was ranked second in the 2013 IEEE Marconi paper awards in wireless communications. His main research interests include cloud radio access networks, cross-layer optimization, cooperative networks, convex optimization, distributed algorithms, and free-space optical communications.

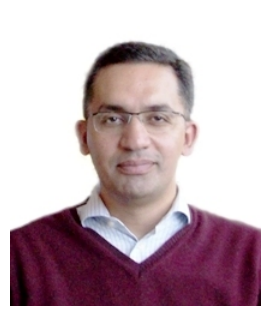

Tareq Y. Al-Naffouri (M'10) received the B.S. degrees in mathematics and electrical engineering (with first honors) from King Fahd University of Petroleum and Minerals, Dhahran, Saudi Arabia, in 1994, the M.S. degree in electrical engineering from the Georgia Institute of Technology, Atlanta, in 1998, and the Ph.D. degree in electrical engineering from Stanford University, Stanford, CA, in 2004. He was a visiting scholar at the California Institute of Technology, Pasadena, from January to August 2005 and during summer 2006. He was a Fulbright scholar at the University of Southern California from February to September 2008. $\mathrm{He}$ has held internship positions at NEC Research Labs, Tokyo, Japan, in 1998, Adaptive Systems Lab, UCLA in 1999, National Semiconductor, Santa Clara, CA, in 2001 and 2002, and Beceem Communications Santa Clara, in 2004. He is currently an Associate Professor with the Electrical Engineering Department, King Fahd University of Petroleum and Minerals, and jointly at the Electrical Engineering Department, King Abdullah University of Science and Technology (KAUST). His research interests lie in the areas of adaptive, statistical, and sparse signal processing and their applications to wireless communications, seismic signal processing, and in multiuser information theory. He has more than 90 publications in Journal and conference proceedings, nine standard contributions, 6 issued patents, and 6 pending.

Dr. Al-Naffouri is the recipient of a 2001 Best Student Paper Award at the IEEE-EURASIP Workshop on Nonlinear Signal and Image Processing (NSIP) 2001 for his work on adaptive filtering analysis, the IEEE Education Society Chapter Achievement Award in 2008, and Al-Marai Award for innovative research in communication in 2009. He has been an Associate Editor of the IEEE Transaction on Signal Processing since August 2013.

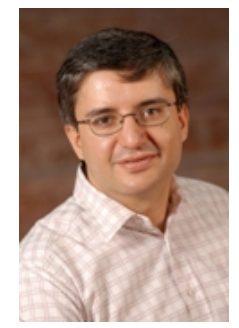

Mohamed-Slim Alouini (S'94-M'98-SM'03-F'09) was born in Tunis, Tunisia. He received the Ph.D. degree in electrical engineering from the California Institute of Technology (Caltech), Pasadena, CA, USA, in 1998. He served as a faculty member at the University of Minnesota, Minneapolis, MN, USA, then in the Texas A\&M University at Qatar, Education City, Doha, Qatar, before joining King Abdullah University of Science and Technology (KAUST), Thuwal, Makkah Province, Saudi Arabia, as a Professor of electrical engineering in 2009. His current research interests include the modeling, design, and performance analysis of wireless communication systems. 